\title{
Analysis of Interconnected Oscillators by Dissipativity Theory
}

\author{
Guy-Bart Stan, Member, IEEE, and Rodolphe Sepulchre
}

\begin{abstract}
This paper employs dissipativity theory for the global analysis of limit cycles in particular dynamical systems of possibly high dimension. Oscillators are regarded as open systems that satisfy a particular dissipation inequality. It is shown that this characterization has implications for the global stability analysis of limit cycle oscillations: i) in isolated oscillators, ii) in interconnections of oscillators, and iii) for the global synchrony analysis in interconnections of identical oscillators.
\end{abstract}

Index Terms-Global limit cycle analysis, global synchronization, Hopf and pitchfork bifurcations, networks of oscillators.

\section{INTRODUCTION}

$\mathbf{O}$ SCILLATORS are dynamical systems that exhibit stable limit cycle oscillations. Models of oscillators abound in biology and in physics (see [1]-[3] and the references therein). Synchrony and phase-locking phenomena in (possibly large) networks of interconnected oscillators are fundamental issues of dynamical system theory and have a wide range of applications (see, e.g., [4]). Nevertheless, because of their nonlinear nature, system theoretic questions about oscillators and networks of oscillators are difficult to address analytically. Following the dissipativity approach introduced by Willems [5], the present paper regards oscillators as open systems, that is, dynamical systems with input $u$ and output $y$, with the objective of addressing system theoretic questions pertaining to interconnections.

Dissipativity theory is based on a characterization of open systems by a dissipation inequality between the storage variation and a supply rate. The storage reflects the energy stored in the internal system components. The supply rate governs the exchange of energy with the external world. The results of this paper build upon a dissipation inequality with a supply rate of the form (if expressed in the single-input-single-output (SISO) framework)

$$
w(u, y)=u y+a_{k}(y)-d(y) \quad a_{k}(y) \geq 0 \quad d(y) \geq 0 .
$$

Manuscript received June 28, 2005; revised March 17, 2006 and June 19, 2006. Recommended by Associate Editor M.-Q. XiaoThe work of the first author was supported by the European Commission under the FP6 Marie-Curie Intra-European Fellowship. This paper presents research partially supported by the Belgian Programme on Inter-University Poles of Attraction, initiated by the Belgian State, Prime Minister's Office for Science, Technology, and Culture.

G.-B. Stan is with the Department of Engineering, Control Group, he University of Cambridge, Cambridge CB2 1PZ, U.K. (e-mail: gvs22@eng.cam.ac.uk).

R. Sepulchre is with the Department of Electrical Engineering and Computer Science, the University of Liège, B-4000 Liège, Belgium (e-mail: r.sepulchre@ulg.ac.be).

Color versions of one or more of the figures in this paper are available online at http://ieeexplore.ieee.org.

Digital Object Identifier 10.1109/TAC.2006.890471

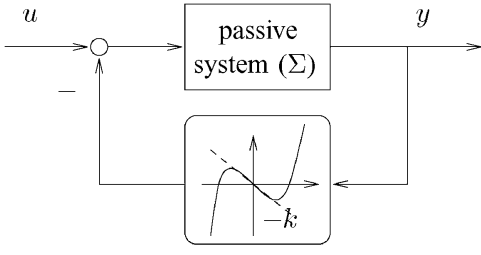

Fig. 1. SISO passive system in feedback with a static nonlinearity $\phi_{k}(\cdot)$ characterized by a parameterized negative slope $(-k)$ at the origin results in dissipativity with respect to the supply rate (1). $u$ (respectively, $y$ ) denotes the external input (respectively, output) of the SISO feedback system.

Without the positive term $a_{k}(y)$, the supply rate (1) is the supply rate of (strictly) passive systems, which plays a fundamental role in the stability analysis of interconnected equilibrium systems (see, for instance, [6] and [7]). The role of the activation term $a_{k}(y)$ in (1) is to revert the sign of dissipation when the output is small. The competition between passive elements (reflected in $u y-d(y))$ that dissipate the storage and active elements (reflected in $a_{k}(y)$ ) that restore the storage constitutes the intuitive basis of the oscillation mechanism. This qualitative description of oscillators was previously advocated by Chua in [8]. From an energetic point of view, passivity w.r.t. the supply rate (1) defines a system that restores energy at low energy, that is, $a_{k}(y)-d(y)>0$ when $|y|$ is small, and that dissipates energy at high energy, that is, $a_{k}(y)-d(y)<0$ when $|y|$ is large.

The simplest way to obtain dissipativity with a supply rate of the form (1) is to consider a SISO passive system $\Sigma$ in feedback with a parametric static nonlinearity $\phi_{k}(\cdot)$ as illustrated in Fig. 1. The static nonlinearity $\phi_{k}(\cdot)$ is defined by $\phi_{k}(y)=$ $-k y+\phi(y)$ which yields the supply rate

$$
w(u, y)=u y+k y^{2}-y \phi(y) .
$$

The parameter $k$ appearing in the definition of $\phi_{k}(\cdot)$ controls the negative slope at the origin and provides a basic bifurcation mechanism to create sustained oscillations in the feedback system as we will see in Section III. The precise assumptions on $\phi(\cdot)$ are postponed to Section II but the reader may think of $\phi(\cdot)$ as a cubic nonlinearity to fix the ideas.

As an extension of the results previously presented in [9] for SISO Lure feedback systems, the first part of this paper (Sections III, IV, and V) provides sufficient conditions for global asymptotic convergence to a limit cycle in a generalized, multiple-input-multiple-output (MIMO) version of the Lure feedback system represented in Fig. 1. As will be shown, a global limit cycle in such MIMO systems either results from a supercritical Hopf bifurcation, or from a supercritical pitchfork bifurcation that yields a globally bistable system which is then easily 
turned into a relaxation oscillation. The first scenario provides a generalization of the Van der Pol oscillators (see, e.g., [10]). Its energy interpretation fits the qualitative description of a lossless exchange of energy between two storage elements, regulated by a locally active but globally dissipative element. The second scenario provides a generalization of Fitzhugh-Nagumo oscillators (see, e.g., [11]). Its energy interpretation fits the qualitative description of many oscillation mechanisms in biology, viewed as periodic switches between two quasi-steady-states.

Such global limit cycle oscillators are named "passive oscillators" since they are dissipative w.r.t. the supply rate (1). An advantage of the proposed dissipativity approach is that it allows to study global limit cycle oscillations in passive oscillators of arbitrary dimension. To illustrate this, a nontrivial (dimension 3) example of passive oscillator is provided in Section VI. Another advantage of the presented approach is that the limit cycle global convergence results that hold for a single passive oscillator extend to networks of interconnected oscillators. This is illustrated in Section VII where we provide limit cycle global convergence results for passive interconnections of passive oscillators.

Beyond limit cycle global convergence, synchronization among interconnected oscillators is an important issue in biological and physical phenomena. Section VIII provides synchronization results for networks of oscillators that satisfy an incremental form of the dissipation inequality (1). Both the dissipation inequality (1) and its incremental form are shown to hold for a specific class of passive oscillators. In Section IX we discuss the required incremental dissipativity conditions from the point of view of graph theory and deduce generic topological coupling condition for synchronization. Finally, Section X concludes and presents some future research topics.

To the best of the authors knowledge, the use of dissipativity theory for the system analysis of interconnected oscillators is new. Many earlier results in the literature have nevertheless exploited the structure of Lure systems in the study of nonlinear oscillations. In [12] and [13], Yakubovich and Tomberg provide sufficient conditions for the existence of sustained oscillations (not necessarily corresponding to a periodic orbit) and this theory has been followed by many developments summarized in [14]. In [15], Mees provides a graphical criterion for Hopf bifurcation in Lure systems. Recently, the authors of [16] have developed novel numerical tools for the global analysis of limit cycles in piecewise linear systems. The use of these numerical tools in our context (restricting to a linear element in the forward path and to a piecewise linear static element in the feedback path) is discussed in [17] and [18].

\section{PRELIMINARIES}

\section{A. Notations and Terminology}

Throughout this paper, we use the following notations. $I_{N}$ denotes the $N \times N$ identity matrix and 1 the column vector $(1, \ldots, 1)^{T} \in \mathbb{R}^{N}$. The Euclidean norm in $\mathbb{R}^{n}$ is denoted as $|\cdot|$, i.e., $|x|^{2}=x^{T} x$ where ${ }^{T}$ defines transposition. $(i) \bmod (N)$ denotes the modulo operation, i.e., integer $i$ is taken modulo $N$. The notation $A \otimes B$ denotes the Kronecker product between the matrices $A$ and $B$ (see [19]). Finally, we say that a real matrix $A$ is positive definite if and only if $x^{T} A x>0$ for all

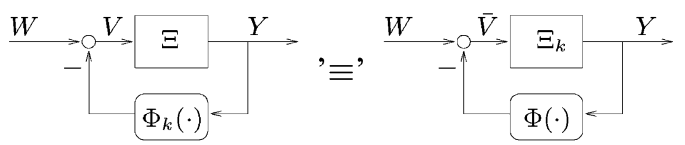

Fig. 2. Equivalent representations of the Lure MIMO nonlinear system studied in this paper. $\Phi_{k}(Y)=\left(\phi_{k}\left(y_{1}\right), \ldots, \phi_{k}\left(y_{N}\right)\right)^{T}$ is a MIMO repeated nonlinearity.

$x \in \mathbb{R}^{n} \backslash\{0\}$. For real positive-semidefinite matrices, the same definition holds except that the inequality is nonstrict. As a consequence of these definitions, a real matrix $A$ is positive (semi-) definite if and only if its symmetric part, denoted by $A_{s}$, is positive (semi-) definite.

\section{B. Lure Feedback Systems}

We consider the Lure system shown in Fig. 2 which represents the MIMO nonlinear system $\Xi$ in feedback with a static nonlinearity $\Phi_{k}(\cdot)$. This figure is a MIMO generalization of the SISO system represented in Fig. 1.

The (square MIMO) system $\Xi$ is described by the state-space model

$$
\Xi\left\{\begin{array}{l}
\dot{X}=f(X)+g(X) V \quad X \in \mathbb{R}^{n} \quad V \in \mathbb{R}^{N} \\
Y=h(X) \quad Y \in \mathbb{R}^{N}
\end{array}\right.
$$

where the vector fields $f(\cdot), g(\cdot)$, and the function $h(\cdot)$ are smooth. We assume that the origin $X=0$ is an equilibrium point of the free system $(V=0)$, i.e., $f(0)=0$, and that $h(0)=0$ and $g(0) \neq 0$. We also assume zero-state detectability of the pair $(f, h)$, i.e., that every solution $X(t)$ of the free system $\dot{X}=f(X)$ that verifies $Y(t)=h(X(t)) \equiv 0$ asymptotically converges to the zero solution $X=0$ as $t \rightarrow \infty$.

We denote by $\Xi_{k}$ the positive feedback interconnection of the system $\Xi$ with the static gain $k I_{N}$, that is, (3) with $V=$ $k Y+\bar{V}$ where $\bar{V}$ denotes the input of $\Xi_{k}$. We denote by $R_{k}(s)$ the MIMO transfer function of the linearization of $\Xi_{k}$ at $X=0$.

The static nonlinearity $\Phi_{k}(\cdot)$ is a MIMO repeated nonlinearity, i.e., $\Phi_{k}(Y)=\left(\phi_{k}\left(y_{1}\right), \ldots, \phi_{k}\left(y_{N}\right)\right)^{T}$ where

$$
\phi_{k}\left(y_{i}\right)=-k y_{i}+\phi\left(y_{i}\right) \quad \forall i=1, \ldots, N
$$

and $y_{i}$ is the $i$ th component of the output vector $Y$. To emphasize the fact that this MIMO nonlinearity is repeated we denote it by $\operatorname{diag}\left\{\phi_{k}(\cdot)\right\}$. The nonlinearity $\phi(\cdot)$ is a smooth sector nonlinearity in the sector $(0, \infty)$. Without loss of generality, we pose $\phi^{\prime}(0)=0$ such that the local slope of $\phi_{k}(\cdot)$ is determined by $k$. The parameter $k$ thus regulates the level of "activation" of the nonlinearity near $X=0$. The sector condition then imposes $\phi^{\prime \prime}(0)=0$. In addition, we assume $\phi^{\prime \prime \prime}(0)=\kappa>0$ and $\lim _{|s| \rightarrow \infty}(\phi(s) / s)=+\infty$. The last condition is known as the "stiffening" nonlinearity condition. It is imposed to facilitate the global analysis of the feedback system (see [20]).

Fig. 2 illustrates two block-diagram representations of the same feedback system with external input $W \in \mathbb{R}^{N}$ : The system $\Xi$ with the feedback interconnection

$$
V=-\Phi_{k}(Y)+W
$$

or the system $\Xi_{k}$ with the feedback interconnection

$$
\bar{V}=-\Phi(Y)+W .
$$




\section{Dissipativity and Strong Passivity}

Dissipativity theory has emerged as a central tool for the stability analysis of feedback systems (see [21], [6], and [7]). For the sake of completeness we recall here the definition of dissipativity as introduced by in [21] and to which the reader is referred for more details. The (state-space) system $\Xi$ with input vector $V$ and output vector $Y$ is dissipative if there exists a scalar storage function $S(X) \geq 0$ and a scalar supply rate $w(V, Y)$ such that the dissipation inequality

$$
S\left(X\left(T^{*}\right)\right)-S(X(0)) \leq \int_{0}^{T^{*}} w(V(t), Y(t)) d t
$$

is satisfied for all $T^{*} \geq 0$ and along any solution $X(t)$ of (3). Passivity is dissipativity with the supply rate $w(V, Y)=$ $V^{T} Y$. Strict output passivity is dissipativity with the supply rate $w(V, Y)=V^{T} Y-d(Y)$, with $d(Y)>0$ for $Y \neq 0$. Similarly, strict input passivity is dissipativity with the supply rate $w(V, Y)=V^{T} Y-e(V)$, with $e(V)>0$ for $V \neq 0$. In the particular case of linear detectable systems, passivity of $\Xi$ is equivalent to positive realness of its associated MIMO transfer function $R(s)$ (see [10, Sec. 6.3]).

If the storage function $S(X)$ is differentiable, the dissipation inequality (7) is equivalently written as

$$
\dot{S}(X(t)) \leq w(V(t), Y(t))
$$

Throughout this paper, we assume additional properties for the storage function $S(X)$.

1) (Smoothness): $S(X)$ is continuously differentiable $\left(\mathcal{C}^{1}\right)$ in $\mathbb{R}^{n}$ and twice continuously differentiable $\left(\mathcal{C}^{2}\right)$ in a neighborhood of the origin.

2) (Lyapunov): $S(X)$ is positive definite (i.e., $S(0)=$ $0, S(X)>0 \forall X \neq 0$ ) and radially unbounded (i.e., $S(X) \rightarrow \infty$ as $|X| \rightarrow \infty)$.

3) (Locally quadratic): The Hessian of $S(X)$ evaluated at zero, i.e., $\left.\left(\partial^{2} S(X) / \partial X^{2}\right)\right|_{X=0}$, is a symmetric positive-definite matrix $P=P^{T}>0$.

To emphasize these extra requirements on the storage function, we say that $\Xi$ is strongly passive whenever $\Xi$ is passive with a storage function that satisfies the three additional assumptions 1)-3). These assumptions are always satisfied in the (detectable) linear case because linear passive systems have quadratic storage functions [21]. More generally, these assumptions are convenient to link the passivity of $\Xi$ to the stability properties of the zero input system since $S(X)$ then serves as a (global) Lyapunov function. The locally quadratic assumption further ensures that the linearization of $\Xi$ is passive, with the quadratic approximation of $S(X)$ as a storage function. It also implies that the system has a relative degree one, i.e., $(\partial h(X) / \partial X) g(X)>0$, for all $X$ in a neighborhood of the origin $X=0$, and that it is weakly minimum phase, i.e., its zero dynamics are Lyapunov stable (see [22]).

\section{Absolute Stability and Multipliers}

The feedback system (3), (4), (5) with $W \equiv 0$ is absolutely stable when the system possesses a unique equilibrium $X=$ 0 which is globally asymptotically stable for any MIMO repeated nonlinearity $\Phi(\cdot)=\operatorname{diag}\{\phi(\cdot)\}$ with $\phi(\cdot)$ in the sector $(0,+\infty)$. Because the static nonlinearity $\Phi(Y)$ is strictly input passive (see [10]), a well-known sufficient condition for absolute stability is that $\Xi_{k}$ is strongly passive and zero-state detectable (see [10] and [7]). Indeed, under such condition, the storage function of $\Xi_{k}, S_{k}(X)$ (where the indice $k$ of the storage function is used to emphasize its dependence on $k$ ), then satisfies the dissipation inequality

$$
\dot{S}_{k} \leq-Y^{T} \Phi(Y)
$$

As a consequence, we may use $S_{k}(X)$ as a global Lyapunov function. Global asymptotic stability of the equilibrium $X=0$ directly follows from the LaSalle invariance principle [7]. Obviously, since $S_{k}(X)$ depends on $k$, absolute stability of the Lure feedback system (3)-(5) will also depend on $k$. Stability analysis w.r.t. $k$ will be discussed in Section III.

The theory of multipliers (see [23]-[25]) provides relaxed conditions for absolute stability. Assume that $H_{1}(s)$ and $H_{2}(s)$ are two SISO transfer functions with both poles and zeros in the left-half plane. Consider the system resulting from the unforced (no external input) feedback interconnection of $\tilde{\Xi}_{k}=$ $H_{1}(s) I_{N} \Xi_{k} H_{2}^{-1}(s) I_{N}$ and $\tilde{\Phi}=H_{2}(s) I_{N} \Phi H_{1}^{-1}(s) I_{N}$ (see [24] for a block diagram interpretation). If $H_{1}(s)$ and $H_{2}(s)$ are such that $\tilde{\Phi}$ is strictly input passive, then strong passivity and zero-state detectability of $\tilde{\Xi}_{k}$ imply the absolute stability of the new feedback system. But stability of the unforced $(W=0)$ feedback interconnection of $\Xi_{k}$ with $\Phi$ is equivalent to stability of the unforced feedback interconnection of $\tilde{\Xi}_{k}$ with $\tilde{\Phi}$, which suggests why the multipliers $H_{1}(s)$ and $H_{2}(s)$ may provide relaxed conditions for the absolute stability of the original unforced feedback interconnection of $\Xi$ with $\Phi_{k}$ (see [24]).

For static nonlinearities $\Phi(\cdot)=\operatorname{diag}\{\phi(\cdot)\}$ respecting the assumptions of Section II-B, the simplest example of multiplier is the Popov multiplier for which $H_{1}(s)=(1+\gamma s), \gamma>0$ and $H_{2}(s)=1$ (see [26]). In this case, requiring strong passivity (and zero-state detectability) of the system $(1+\gamma s) I_{N} \Xi_{k}$ for absolute stability of the feedback system (3), (4), (5) with $W \equiv$ 0 defines the Popov criterion (see [27]).

For static nonlinearities $\Phi(\cdot)=\operatorname{diag}\{\phi(\cdot)\}$ respecting the assumptions of Section II-B and such that $\phi(\cdot)$ is furthermore monotone increasing, a broad class of multipliers was introduced in [23] by Zames and Falb in the form

$$
\begin{aligned}
& M(j \omega)=1-Z(j \omega)=1-\int_{-\infty}^{+\infty} z(t) e^{-j \omega t} d t \\
& \int_{-\infty}^{+\infty}|z(t)| d t<1 .
\end{aligned}
$$

The additional assumption $z(t) \geq 0$ is also needed unless $\phi(\cdot)$ is odd. Zames and Falb showed that multipliers of the form (8), which are not necessarily causal, can always be factored in the form

$$
M(s)=H_{1}(s) H_{2}(-s)
$$

with $H_{1}(s), H_{2}(s)$, and their inverses being causal and stable and with $\tilde{\Phi}$ being strictly input passive (see [23] for the SISO case and [24] for its MIMO generalization). As a consequence, strong passivity and zero-state detectability of $\tilde{\Xi}_{k}$ is sufficient 
for absolute stability of the feedback system (3)-(5) with $W \equiv$ 0 . Note that when $\Xi_{k}$ is a linear system, passivity of $\tilde{\Xi}_{k}$ is equivalent to positive realness of its associated transfer function $H_{1}(s) I_{N} R_{k}(s) H_{2}^{-1}(s) I_{N}$.

For later reference, we summarize in Theorem 1 sufficient conditions for absolute stability of the unforced $(W \equiv 0)$ MIMO Lure feedback system represented in Fig. 2. In Theorem 1 , we assume that the feedback interconnection is ultimately bounded which means that all solutions enter, in finite time, a compact and invariant set $\Omega=\Omega(k)$ (see [10, Def. 5.1]).

Theorem 1: Consider the feedback system (3)-(5) with $W \equiv$ 0 and $k$ fixed to a particular value. If $\Xi$ and its linearization are zero-state detectable and the feedback interconnection of $\Xi$ and $\Phi_{k}(\cdot)$ is ultimately bounded, then each of the following conditions is sufficient for global asymptotic stability of the equilibrium $X=0$ of the feedback system.

- $\phi(\cdot)$ is in the the sector $(0, \infty)$ and there exists $\gamma>0$ such that $(1+\gamma s) I_{N} \Xi_{k}$ is strongly passive.

- $\phi(\cdot)$ is monotone increasing and in the sector $(0, \infty)$, and there exists $M(s)=H_{1}(s) H_{2}(-s)$ with $M(s)$ in the form (8) and $z(t) \geq 0$, such that $\tilde{\Xi}_{k}=H_{1}(s) I_{N} \Xi_{k} H_{2}^{-1}(s) I_{N}$ is strongly passive.

- $\phi(\cdot)$ is odd, monotone increasing and in the sector $(0, \infty)$, and there exists $M(s)=H_{1}(s) H_{2}(-s)$ with $M(s)$ in the form (8) such that $\tilde{\Xi}_{k}=H_{1}(s) I_{N} \Xi_{k} H_{2}^{-1}(s) I_{N}$ is strongly passive.

The proof of Theorem 1 is given in [9] in the SISO case. The extension of this proof to the MIMO case is straightforward.

Remark 1: A MIMO extension of Arcak's results in [20] shows that the unforced feedback system (3), (4), (5) is ultimately bounded for any $k<\infty$ if $\Xi$ is linear, passive, zero-state detectable and $\Phi_{k}(\cdot)$ is a MIMO repeated nonlinearity, i.e., $\Phi_{k}(\cdot)=\operatorname{diag}\left\{\phi_{k}(\cdot)\right\}$, with $\phi(\cdot)$ satisfying the assumptions of Section II-B and monotone increasing. Details of this extension are omitted here but can be found in [18].

\section{Bifurcations in Absolutely Stable FEEDBACK SYSTEMS}

In this section, we analyze the stability properties of the unforced $(W \equiv 0)$ feedback system (3)-(5) as the parameter $k$ increases from 0 . Throughout the rest of the paper, the notation $k \gtrsim k^{*}$ is used to denote a value of the parameter $k$ slightly greater than the critical bifurcation value $k^{*}$, i.e., $k \in\left(k^{*}, \bar{k}\right]$ for some $\bar{k}>k^{*}$. Since we assume that $\Xi$ is strongly passive and zero-state detectable, the feedback system (3)-(5) with $W \equiv 0$ is absolutely stable for $k=0$. However, it can be showed that a bifurcation necessarily occurs when $k$ is increased from 0 because the linearization of this feedback system at $X=0$ possesses at least one eigenvalue in the right half plane when $k$ becomes large enough (see [18] for more details based on a simple root locus argument). Let $k^{*}(\geq 0)$ denote the smallest value of $k$ at which asymptotic stability of the linearized system at $X=0$ is lost. The two following examples illustrate in their simplest form the two bifurcation scenarii that generically occur when the value of $k$ is increased beyond $k^{*}$.

Example 1: Consider the unforced SISO feedback system (3)-(5) in the particular case where $\Xi$ is a pure integrator with associated transfer function $R(s)=(1 / s)$ and $\phi_{k}(y)=-k y+$ $y^{3}$. The state representation of the feedback system is

$$
\dot{y}=k y-y^{3}
$$

which is the normal form of a supercritical pitchfork bifurcation: $y=0$ is asymptotically stable for $k \leq k^{*}=0$ while for $k>k^{*}, y=0$ is unstable and two stable equilibria appear.

Example 2: Consider the same unforced feedback system as in Example 1 but where $\Xi$ is a linear system with associated transfer function $R(s)=\left(s / s^{2}+1\right)$. The dynamics of the feedback system is then governed by

$$
\ddot{y}+y+\frac{d}{d t}\left(y^{3}-k y\right)=0
$$

which is a standard form of the Van der Pol oscillator. For $k \leq$ $k^{*}=0$, the equilibrium $y=0$ is globally asymptotically stable. At $k=k^{*}$, a supercritical Hopf bifurcation occurs, that is, two complex eigenvalues cross the imaginary axis. For $k>k^{*}, y=0$ is unstable and all other solutions converge to a unique asymptotically stable limit cycle.

In the two examples shown previously, the bifurcation is supercritical and the global convergence of the solutions to a neighborhood of the equilibrium $X=0$ is not destroyed in the vicinity of the bifurcation. We interpret these two properties as resulting from passivity of the transfer function $R_{k^{*}}(s)$ at the bifurcation point. The following result generalizes these two bifurcation scenarii.

Theorem 2: Consider the feedback system (3), (4), (5) with $W \equiv 0$. Assume that $\Xi$ is strongly passive, that both $\Xi$ and its linearization are zero-state detectable, and that, for the values of $k$ considered in Case (1) and Case (2), the feedback interconnection of $\Xi$ and $\Phi_{k}(\cdot)$ is ultimately bounded. Let $k^{*} \geq 0$ be the smallest value of $k$ at which the corresponding MIMO transfer function $R_{k^{*}}(s)$ has a pole on the imaginary axis. If $\Xi_{k^{*}}$ is strongly passive and

Case 1): If $R_{k^{*}}(s)$ has a unique pole on the imaginary axis, then the bifurcation is a supercritical pitchfork bifurcation such that, for $k \gtrsim k^{*}$, the system is globally bistable, i.e., the equilibrium point $X=0$ is a saddle and its stable manifold $E_{s}(0)$ separates the state space in two open sets, each of which is the basin of attraction of a stable equilibrium point.

Case 2): If $R_{k^{*}}(s)$ has a unique pair of conjugated poles on the imaginary axis, then the bifurcation is a supercritical Hopf bifurcation such that, for $k \gtrsim k^{*}$, the system is characterized by a unique limit cycle which is globally asymptotically stable in $\mathbb{R}^{n} \backslash E_{s}(0)$ where $E_{s}(0)$ denotes the stable manifold of the unstable equilibrium $X=0$.

Proof: The proof is divided into a local argument and a global argument. Both arguments rely on the dissipation inequality of the unforced system $(W \equiv 0)$ at the bifurcation point $\left(k=k^{*}\right)$

$$
\dot{S}_{k^{*}} \leq-Y^{T} \Phi(Y)
$$

where $S_{k^{*}}(X)$ denotes the storage function of $\Xi_{k^{*}}$. The local argument will show the existence of a supercritical Hopf (respectively, pitchfork) bifurcation at $\epsilon=k-k^{*}=0$. This implies the existence of a constant $\bar{\epsilon}_{1}>0$ and a neighborhood 
$\mathcal{U} \subset \mathbb{R}^{n}$ of $X=0$ such that for each $\epsilon \in\left(0, \bar{\epsilon}_{1}\right]$, all solutions with initial condition in $\mathcal{U}$ either converge to the unstable equilibrium $X=0$ or to a unique stable limit cycle of radius $\mathcal{O}(\sqrt{\epsilon})$ (respectively, one of the two stable equilibria, each located at a distance $\mathcal{O}(\sqrt{\epsilon})$ of the origin). The global argument will show that there exists a constant $\bar{\epsilon}_{2}>0, \bar{\epsilon}_{2} \leq \bar{\epsilon}_{1}$, such that for each $\epsilon \in\left(0, \bar{\epsilon}_{2}\right]$, all solutions eventually enter the previously defined neighborhood $\mathcal{U}$ in finite time (which means that the local argument eventually applies to each solution).

We first prove the global argument. Ultimate boundedness of the feedback system implies that for each $\epsilon \in\left(0, \bar{\epsilon}_{3}\right]$, all solutions enter in finite time an invariant compact set $\Omega=\Omega(\epsilon)$. Global asymptotic stability of $X=0$ at $\epsilon=0$ implies practical semiglobal stability of the solution $X=0$ for small $\epsilon>0$, that is, for any given neighborhood $\mathcal{U}$, there always exists an $\bar{\epsilon}_{2} \leq \bar{\epsilon}_{3}$ such that, for each $\epsilon \in\left(0, \bar{\epsilon}_{2}\right]$, all solutions with initial condition in $\Omega(\epsilon)$ enter $\mathcal{U}$ in finite time (see [28] for a definition of practical semiglobal stability and the necessary conditions for it).

Next we turn to the local argument. At the bifurcation, i.e., at $k=k^{*}$, the system possesses a center manifold (see [29]). Detectability of the linearization of $\Xi$ implies observability of the linearized center manifold dynamics. From (9) and the definition of $\Phi(\cdot)$ [see also (4)], we can write, locally around $X=0$

$$
\dot{S}_{k^{*}} \leq-\kappa \sum_{j=1}^{N} y_{j}^{4}+\mathcal{O}\left(|Y|^{5}\right), \quad \kappa>0 .
$$

Case 1) (one-dimensional center manifold): If $R_{k^{*}}(s)$ has a unique pole on the imaginary axis, the center manifold is onedimensional. The normal form of the center manifold dynamics writes (see [29])

$$
\dot{\xi}=a_{3} \xi^{3}+\mathcal{O}\left(\xi^{4}\right), \quad \xi \in \mathbb{R} .
$$

The restriction of $S_{k^{*}}(X)$ on the center manifold is a locally quadratic function of the form $S_{\mathrm{cm}}(\xi)=(1 / 2) P_{1} \xi^{2}+\mathcal{O}\left(\xi^{3}\right)$ (with $P_{1}>0$ from the strong passivity assumption of $\Xi_{k^{*}}$ ) that satisfies the dissipation inequality

$$
\dot{S}_{\mathrm{cm}}=P_{1} \xi \dot{\xi} \leq-\kappa \sum_{j} y_{j}^{4}+\mathcal{O}\left(|Y|^{5}\right) .
$$

In the center manifold, each output component writes $y_{i}=$ $c_{i} \xi+\mathcal{O}\left(|\xi|^{2}\right)$. Observability of the linearized center manifold dynamics implies that $c_{i} \neq 0$ for at least one value of $i \in$ $\{1, \ldots, N\}$. This forces $a_{3}<0$ in (11). The bifurcation is thus a supercritical pitchfork, i.e., for $\epsilon \gtrsim 0$, all solutions in $\mathcal{U}$ converge to the unstable equilibrium point $X=0$ or to one of the two asymptotically stable equilibria located at a distance $\pm \mathcal{O}(\sqrt{\epsilon})$ of $X=0$.

Case 2) (two-dimensional center manifold): If $R_{k^{*}}(s)$ has two conjugated poles at $s= \pm j \omega$, the center manifold is two-dimensional. The normal form of the center manifold dynamics is (see [29])

$$
\begin{aligned}
\dot{\xi} & =A_{c} \xi+|\xi|^{2}\left(\begin{array}{l}
a_{3} \xi_{1}-b_{3} \xi_{2} \\
b_{3} \xi_{1}+a_{3} \xi_{2}
\end{array}\right)+\mathcal{O}\left(|\xi|^{4}\right) \\
A_{c} & =\left(\begin{array}{cc}
0 & \omega \\
-\omega & 0
\end{array}\right),
\end{aligned}
$$

which, in polar coordinates, yields

$$
\begin{aligned}
& \dot{\rho}=a_{3} \rho^{3}+\mathcal{O}\left(\rho^{4}\right) \\
& \dot{\theta}=\omega+\mathcal{O}\left(\rho^{2}\right) .
\end{aligned}
$$

The restriction of $S_{k^{*}}(X)$ on the center manifold is a locally quadratic function of the form $S_{\mathrm{cm}}(\xi)=\xi^{T} Q \xi+\mathcal{O}\left(|\xi|^{3}\right)$ with $Q=Q^{T}>0$, that satisfies

$$
\begin{aligned}
\dot{S}_{\mathrm{cm}} & =\xi^{T}\left(Q A_{c}+A_{c}^{T} Q\right) \xi+\mathcal{O}\left(|\xi|^{3}\right) \\
& \leq-\kappa \sum_{j=1}^{N} y_{j}^{4}+\mathcal{O}\left(|Y|^{5}\right) .
\end{aligned}
$$

Up to a scaling factor, the only symmetric, positive-definite solution $Q$ of $Q A_{c}+A_{c}^{T} Q \leq 0$ is $Q=(1 / 2) I$, which implies $S_{\mathrm{cm}}(\rho)=(1 / 2) \rho^{2}+\mathcal{O}\left(\rho^{3}\right)$. For initial conditions in the center manifold, the dissipation inequality (15) thus satisfies

$$
\dot{S}_{\mathrm{cm}}=a_{3} \rho^{4}+\mathcal{O}\left(\rho^{5}\right) \leq-\kappa \sum_{j=1}^{N} y_{j}^{4}+\mathcal{O}\left(|Y|^{5}\right) .
$$

Integration on both sides over an arbitrarily chosen time interval $T^{*}>0$ yields

$$
a_{3} \int_{0}^{T^{*}}(\rho(t))^{4} d t+\mathcal{O}\left(\rho^{5}\right) \leq-\kappa \int_{0}^{T^{*}} \sum_{j=1}^{N}\left(y_{j}(t)\right)^{4} d t+\mathcal{O}\left(|Y|^{5}\right)
$$

which, from the observability of the linearized center manifold dynamics, forces $a_{3}<0$. This implies that the bifurcation is a supercritical Hopf bifurcation, that is, for $\epsilon \gtrsim 0$, all solutions in $\mathcal{U}$ either converge to the unstable equilibrium $X=0$ or to a unique stable limit cycle of radius $\mathcal{O}(\sqrt{\epsilon})$. This concludes the proof.

We briefly comment on the technical assumptions of Theorem 2 : the detectability assumption is a natural assumption in a context where (internal) stability is deduced from an (external) passivity property; as in Theorem 1, the ultimate boundedness assumption allows for global conclusions; finally, the restriction to one or two eigenvalues on the imaginary axis at the bifurcation excludes degenerate bifurcations.

The central assumption of Theorem 2 is that $\Xi_{k^{*}}$ is strongly passive. This assumption is rather restrictive. As the parameter $k$ increases, $\Xi_{k}$ loses passivity at $k=k_{\text {passive }}^{*}$ and it loses stability at $k=k^{*}$. One necessarily has $k_{\text {passive }}^{*} \leq k^{*}$, but the passivity assumption on $\Xi_{k^{*}}$ requires $k_{\text {passive }}^{*}=k^{*}$. This assumption can be weakened through the use of multipliers as shown in the following result.

Theorem 3: The statements of Theorem 2 hold if the strong passivity assumption on $\Xi_{k^{*}}$ is replaced by one of the three relaxed conditions of Theorem 1 expressed at $k=k^{*}$.

Proof: The global argument of the proof of Theorem 2 is unchanged because it relies on absolute stability of the system when $\epsilon=k-k^{*}=0$. As a consequence of Theorem 1, conditions of Theorem 3 still guarantee absolute stability when $\epsilon=0$. For the local argument, in the case of Popov multipliers, the dissipation inequality (9) is recovered with the new storage $\mathcal{S}_{k^{*}}=S_{k^{*}}+\gamma \sum_{i=1}^{N} \int_{0}^{y_{i}} \phi(s) d s$. In the case of Zames-Falb multipliers, let $\tilde{S}_{k^{*}}$ be the storage function associated with $\tilde{\Xi}_{k^{*}}$. 
Since, by assumption, $\tilde{\Xi}_{k^{*}}$ is strongly passive, $\tilde{S}_{k^{*}}$ satisfies the dissipation inequality

$$
\dot{\tilde{S}}_{k^{*}} \leq \tilde{V}^{T} \tilde{Y}
$$

with $\tilde{Y}=H_{1}(s) I_{N} Y$ and $\tilde{V}=-H_{2}(s) I_{N} \Phi(Y)=-\tilde{\Phi}(\tilde{Y})$. A minimal realization of the operator $(-\tilde{\Phi})$ is of the form

$$
(-\tilde{\Phi}) \begin{cases}\dot{w}_{1}=A_{1} w_{1}+B_{1} \tilde{Y}, & Y=C_{1} w_{1}+D_{1} \tilde{Y} \\ \dot{w}_{2}=A_{2} w_{2}+B_{2} \Phi(Y), & \tilde{V}=-C_{2} w_{2}-D_{2} \Phi(Y)\end{cases}
$$

with $\left(A_{i}, B_{i}, C_{i}, D_{i}\right),(i=1,2)$, being minimal realization of the linear operators $H_{1}^{-1}$ and $H_{2}$, respectively. From the assumptions of Zames and Falb (see [23]), the linear operators $H_{1}$ and $H_{2}$ are invertible and $H_{1}, H_{1}^{-1}, H_{2}$ and $H_{2}^{-1}$ are causal and bounded (i.e., have finite gains). This implies that the associated transfer functions $H_{1}(s)$ and $H_{2}(s)$ have all their poles and zeros in the open left half plane and thus that the filters $H_{1}(s) I_{N}, H_{1}^{-1}(s) I_{N}, H_{2}(s) I_{N}$ and $H_{2}^{-1}(s) I_{N}$ do not change the dimension of the center manifold. Thus, similarly to the proof of Theorem 2, the center manifold dynamics, expressed in normal form, take the expression (11) when $R_{k^{*}}(s)$ has a unique pole at $s=0$, and the expression (13) when $R_{k^{*}}(s)$ has two conjugated poles at $s= \pm j \omega$.

In order to analyze the dissipation inequality (16) on the center manifold, we approximate the expressions of $\tilde{V}, w_{2}, \tilde{Y}$, and $w_{1}$ as functions of $\xi$ up to suitable order. We use the notation $a^{(n)}(\xi)$ to denote the series expansion of $a(\xi)$, in terms of $\xi$, up to order $n$. If $a^{n}(\xi)$ is a vector function the notation means a component-wise series expansion up to order $n$ for each component of $a(\xi)$. Using this notation, we consider: $\tilde{V}=\tilde{V}^{(3)}(\xi)+\mathcal{O}\left(|\xi|^{4}\right), w_{2}=h_{2}^{(3)}(\xi)+\mathcal{O}\left(|\xi|^{4}\right), \tilde{Y}=$ $\tilde{C} \xi+\mathcal{O}\left(|\xi|^{2}\right)$, and $w_{1}=h_{1} \xi+\mathcal{O}\left(|\xi|^{2}\right)$. From (17) and the assumption $\phi^{\prime \prime \prime}(0)=\kappa>0$, we have

$$
\begin{aligned}
\tilde{V}^{(3)}(\xi) & =-C_{2} h_{2}^{(3)}(\xi)-D_{2} \kappa(C \xi)^{3} \\
C & =C_{1} h_{1}+D_{1} \tilde{C}, \quad \kappa>0
\end{aligned}
$$

where the notation $(C \xi)^{3}$ means a component-wise exponential operation on the vector $(C \xi)$. The function $h_{2}^{(3)}(\xi)$ is solution of the partial differential equation that expresses invariance of the center manifold up to terms of order $\mathcal{O}\left(|\xi|^{4}\right)$ (see [30]):

$$
\begin{aligned}
(- & \left.C_{2} \frac{\partial h_{2}^{(3)}(\xi)}{\partial \xi}-D_{2} 3 \kappa(C \xi)^{2} C\right) A_{\mathrm{cm}} \xi \\
= & -C_{2} A_{2} h_{2}^{(3)}(\xi)-C_{2} B_{2} \kappa(C \xi)^{3} \\
& -D_{2} 3 \kappa(C \xi)^{2} C A_{\mathrm{cm}} \xi
\end{aligned}
$$

with the boundary conditions $h_{2}^{(3)}(0)=0,\left(\partial h_{2}^{(3)} / \partial \xi\right)(0)=0$. In (19), $A_{\mathrm{cm}}=0$ when the center manifold is one dimensional, and $A_{\mathrm{cm}}=A_{c}$ (see (13)) when the center manifold is two dimensional. Once the solution $h_{2}^{(3)}(\xi)$ of (19) is found, the expression of $\tilde{V}^{(3)}(\xi)$ is obtained through (18). We do not even need to solve the partial differential equation (19) for $h_{2}^{(3)}(\xi)$ to obtain the corresponding expression for $\tilde{V}^{(3)}(\xi)$ since the solution $\tilde{V}^{(3)}(\xi(t))$ coincides $^{1}$ with the unique steady-state output

\footnotetext{
${ }^{1}$ This is because the partial differential equation (19) satisfied by $h_{2}^{(3)}(\xi(t))$ is the same as the steady-state partial differential equation satisfied by $h_{2}^{(3)}(\xi(t))$ when the input of the nonlinear dynamic operator $\left(-\tilde{\Phi}^{(3)}\right)$ is $\tilde{Y}^{(1)}(\xi(0), t)=$ $\tilde{C} e^{A_{\mathrm{cm}} t} \xi(0)$ (see [31, Ch. 8]).
}

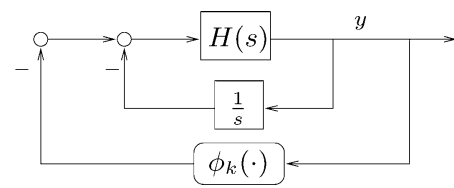

Fig. 3. Forcing the Hopf bifurcation with an integrator in the feedback loop and $H(s)$ passive. The case $H(s)=(1 / s)$ corresponds to Liénard systems.

of the operator $\left(-\tilde{\Phi}^{(3)}\right),{ }^{2}$ when this operator is applied to the (periodic) input $\tilde{Y}^{(1)}(\xi(0), t)=\tilde{C} e^{A_{\mathrm{cm}} t} \xi(0)$ (see ([31, Ch. 8]).

Case 1) (one-dimensional center manifold): When $\xi(=\xi(0)) \in \mathbb{R}$, the constant input $\tilde{Y}^{(1)}=\tilde{C} \xi$ (of the nonlinear dynamic operator $\left(-\tilde{\Phi}^{(3)}\right)$ ) gives rise to the constant output $\tilde{V}^{(3)}(\xi)=\beta \xi^{3}$. Strict input passivity (see [24]) of the operator $\left(-\tilde{\Phi}^{(3)}\right)$ implies that $\tilde{C}^{T} \beta=-\gamma<0$. The dissipation inequality thus becomes

$$
\dot{\tilde{S}}_{k^{*}} \leq\left(\tilde{V}^{(3)}(\xi)\right)^{T} \tilde{Y}^{(1)}(\xi)+\mathcal{O}\left(|\xi|^{5}\right)=-\gamma \xi^{4}+\mathcal{O}\left(|\xi|^{5}\right)
$$

which forces the existence of a supercritical pitchfork bifurcation, as in the proof of Theorem 2.

Case 2) (two-dimensional center manifold): When $\xi(=\xi(0)) \in \mathbb{R}^{2}$, the periodic input $\tilde{Y}^{(1)}(\xi, t)=\tilde{C} e^{A_{c} t} \xi$ (of the nonlinear dynamic operator $\left(-\tilde{\Phi}^{(3)}\right)$ ) gives rise to the periodic output $\tilde{V}^{(3)}(\xi, t)$. Strict passivity and homogeneity of the operator $\left(-\tilde{\Phi}^{(3)}\right)$ implies (see [24])

$$
\begin{aligned}
& \int_{0}^{T^{*}}\left(\tilde{V}^{(3)}(\xi, t)\right)^{T} \tilde{Y}^{(1)}(\xi, t) d t<-\gamma|\xi|^{4}+\mathcal{O}\left(|\xi|^{5}\right), \\
& T^{*}=\frac{2 \pi}{\omega}
\end{aligned}
$$

with $\gamma>0$. For initial conditions in the center manifold, integration of (16) over the period $T^{*}$ leads, locally, to

$$
\tilde{S}_{k^{*}}\left(X\left(T^{*}\right)\right)-\tilde{S}_{k^{*}}(X(0))<-\gamma|\xi|^{4}+\mathcal{O}\left(|\xi|^{5}\right) .
$$

As in the Proof of Theorem 2, this forces $a_{3}<0$ in the center manifold dynamics (14) (see [18]), which proves the existence of a supercritical Hopf bifurcation. This concludes the proof.

The next two sections show that the results presented in Theorem 2 are the basis for two different global feedback oscillation mechanisms.

\section{Hopf BifurCATIONS AND Global OSCILLATIONS}

As mentioned in Example 2, the simplest illustration of the Hopf bifurcation mechanism described in Theorem 2 is provided by the Liénard system

$$
\ddot{y}+y+\frac{d}{d t} \phi_{k}(y)=0, \quad y \in \mathbb{R}
$$

where $\phi_{k}(\cdot)$ satisfies the assumptions made in Section II-B. It admits the feedback representation shown in Fig. 3 when $H(s)=(1 / s)$. In this case, $\Xi$ corresponds to the feedback interconnection of two integrators and its associated transfer function

\footnotetext{
${ }^{2}$ The operator $\left(-\tilde{\Phi}^{(3)}\right)$ corresponds the operator $(-\tilde{\Phi})$ defined in (17) with $\Phi(\cdot)$ replaced by its cubic approximation.
} 
is $R(s)=\left(s / s^{2}+1\right)$. It is well known that the Liénard system (20) has a globally asymptotically stable equilibrium at the origin for $k \leq 0$ and a globally asymptotically stable limit cycle for $k>0$ (see [10]). The result for $k \gtrsim 0$ follows from Theorem 2 because $\Xi$ is the feedback interconnection of two SISO, linear, passive systems (two simple integrators) and because it has two poles on the imaginary axis when $k=0$. Since the (negative) feedback interconnection of two passive systems is still a passive system, Theorem 2 extends this low-order Liénard system result to an arbitrary high-order (strongly) passive system $H(s)$ in feedback with an integrator provided that, at the critical value $k^{*}$ at which the equilibrium $X=0$ loses stability, the corresponding system $\Xi_{k^{*}}$ is passive and zero-state detectable.

The Hopf bifurcation in the feedback system of Fig. 3 has the following energy interpretation: passivity at the bifurcation point allows for a lossless exchange of energy between two storage elements $(H(s)$ and $(1 / s))$. The static nonlinearity $\phi_{k}(\cdot)$ regulates the dissipation in the feedback system, restoring energy when it is too low and dissipating it when it is too high. In the popular Van der Pol oscillator, the two storage elements are a capacitor and an inductor, whereas the dissipation is regulated by means of (for instance) a tunnel-diode circuit, modeled as a static negative resistance whose input-output function is $\phi_{k}(y)=y^{3}-k y$. Theorem 2 extends this feedback mechanism for oscillations to higher dimensional systems.

It should also be observed that, putting an integrator in feedback with an arbitrary $H(s)$ (as in Fig. 3) forces the Hopf bifurcation scenario because of the resulting presence of a zero at $s=0$ in the transfer function $R_{k}(s)=(R(s) / 1-k R(s))=$ $(s H(s) / s+(1-k s) H(s))$ : for the positive feedback interconnection of $R(s)$ with the static gain $k$, the root locus is such that parts of the real axis located at the right of an odd number of singularities (poles or zeros) belong to the root locus. As the transfer function of a strongly passive system, $R(s)$ has a relative degree equal to one and all its poles and zeros belong to the closed left-half plane. As a consequence, the positive part of the real axis necessarily belongs to the root locus and one branch (at least) of the root locus must enter the right-half plane. The presence of a zero at $s=0$ then necessarily implies that (at least) two non-zero eigenvalues cross the imaginary axis at some critical value $k^{*}$ which corresponds to the Hopf bifurcation scenario. Standard Hopf bifurcation is generic, that is, it always happens except in the degenerate case where more than two eigenvalues cross the imaginary axis simultaneously.

\section{Pitchfork Bifurcation, Bistability, and Global RELAXATION OSCILLATIONS}

The pitchfork bifurcation scenario of Theorem 2 is the basis for a second global oscillation mechanism best exemplified with the Fitzhugh-Nagumo model (see [32, Sec. 7.5]). ${ }^{3}$

$$
\begin{aligned}
\dot{y} & =k y-y^{3}-z \\
\tau \dot{z} & =-z+b \tau y
\end{aligned}
$$

${ }^{3}$ The particular (21), (22) are obtained from the Fitzhugh-Nagumo model in [32] with the change of coordinates $y=v-(a+1 / 3), z=w-f(a+1 / 3)-$ $I_{a}$, the definition $\tau=(1 / \gamma)$, and a well-chosen value of the input current $I_{a}$, i.e., $I_{a}=(b / \gamma)(a+1 / 3)-f(a+1 / 3)$. The corresponding value of $k$ is then $k=(1 / 3)\left(a^{2}-a+1\right)>0$

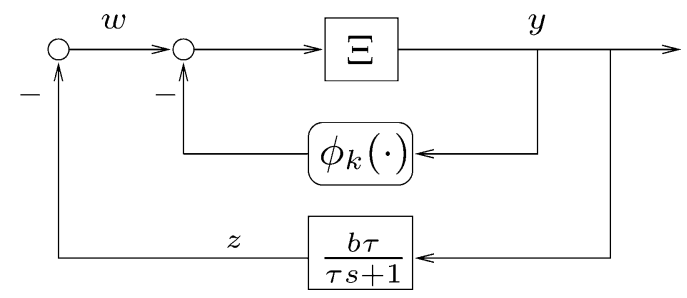

Fig. 4. Converting the pitchfork bifurcation scenario into a relaxation oscillator by adding a slow adaptation mechanism $(\tau \gg 0)$. The case $\Xi=(1 / \mathrm{s})$ corresponds to the Fitzhugh-Nagumo oscillator.

which admits the block diagram representation shown in Fig. 4 with $\Xi=(1 / s)$ and $\phi_{k}(y)=y^{3}-k y$. For $k>0$, the inner-loop

$$
\dot{y}=k y-y^{3}-z
$$

is a globally bistable system over the range of parameter $z \in$ $(-(2 / 3) k \sqrt{(k / 3)},(2 / 3) k \sqrt{(k / 3)})$. Outside of this range, the inner-loop is absolutely stable and has a unique globally asymptotically stable equilibrium. Treating $z$ as a parameter, one thus obtains the bifurcation diagram shown in Fig. 5(a) for $z \in$ $(-(2 / 3) k \sqrt{(k / 3)},(2 / 3) k \sqrt{(k / 3)})$.

The outer-loop in Fig. 4 or equivalently the adaptation dynamics

$$
\tau \dot{z}=-z+b \tau y
$$

combined with the feedback $w=-z$, converts the above described bistable system into a relaxation oscillation in the phase plane $(y, z)$ as shown in Fig. 5(b). The corresponding limit cycle is guaranteed to be globally asymptotically stable provided that the time constant $\tau$ is large enough (see [10]).

Since $b$ plays no particular role in this relaxation oscillation mechanism, we will assume without loss of generality that $b=$ $\tau^{-1}$ in (24), leading to

$$
\tau \dot{z}=-z+y
$$

The global bistability of the inner loop combined with the slow adaptation of the outer loop thus provides a feedback mechanism for global oscillations. The resulting oscillation is a relaxation oscillation characterized by a rapid switch between two quasi-steady-states (i.e., states that would correspond to stable equilibria in the absence of adaptation [10]). Such oscillation mechanisms are frequent in biology (see, e.g., [32]). In the Fitzhugh-Nagumo model, a simplification of Hodgkin-Huxley model for voltage oscillations in the neuron cell membrane, the switch is between the (high) equilibrium potential associated to potassium ions and the (low) equilibrium potential associated to sodium ions. The "recovery" variable $z$ models the voltage dependent opening (closing) of the sodium ion channels and the corresponding closing (opening) of the potassium ion channels (see [11]).

Theorem 2 provides a high-dimensional generalization of the global bistability in the inner loop of Fig. 4. In order to convert the global bistability result of Theorem 2 into a mechanism for global oscillations, we add the scalar adaptation dynamics (25) to the system described in Fig. 2. This is summarized in Theorem 4 where $w_{j}$ represents the $j$ th component of the external input vector $W$ (see Fig. 2). 


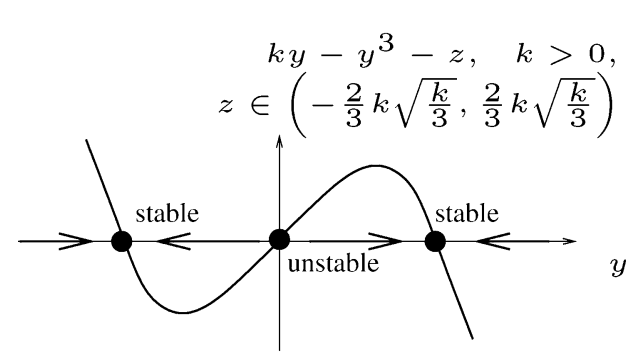

(a)

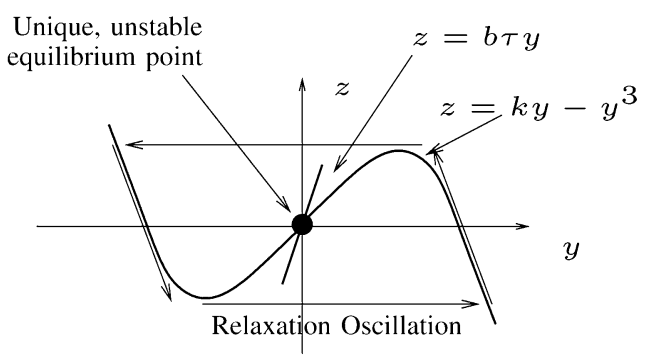

(b)

Fig. 5. Hysteresis associated to a bistable system: (a) without adaptation Globally bistable system; (b) with adaptation Relaxation oscillation.

Theorem 4: Under the assumptions of Theorem 2, suppose that the unforced feedback system (3)-(5) undergoes a supercritical pitchfork bifurcation at $k=k^{*}$. Consider the input $w_{i}=-z, w_{j}=0$ for $j \neq i$ with $z$ satisfying the dynamics $\tau \dot{z}=-z+y_{i}$ and $i$ selected such that the linear center manifold dynamics is observable from $y_{i}$. Assume that the augmented system is ultimately bounded. Then, there exists a positive constant $\bar{\epsilon}$ such that for any particular value of $k$ in $\left(k^{*}, k^{*}+\bar{\epsilon}\right)$, all solutions with initial conditions in $\mathbb{R}^{n+1} \backslash E_{s}(0)$ converge to a unique asymptotically stable limit cycle if $\tau \gg\left(k-k^{*}\right)^{-1}$.

Proof: bAs in the proof of Theorem 2, the reasoning is divided into a local and a global argument. We start with the local argument. Let $\epsilon=\left(k-k^{*}\right)$. By assumption, the unforced feedback system (3)-(5) possesses a one dimensional center manifold at $\epsilon=0$. As shown in the proof of Theorem 2, at the bifurcation point, i.e., at $k=k^{*}$, detectability of the feedback system linearized around $X=0$ implies observability of the linearized center manifold dynamics from at least one output component, e.g., $y_{i}$. As a consequence of the observability of the linearized center manifold dynamics from $y_{i}$, this output component qualifies as a local coordinate in the center manifold and the corresponding center-unstable manifold dynamics can be written

$$
\dot{y}_{i}=\epsilon y_{i}-\kappa^{\prime} y_{i}^{3}+\sum_{j=1}^{N} \alpha_{j} w_{j}+\mathcal{O}\left(y_{i}^{4}\right)
$$

where $\kappa^{\prime}>0$. Observability of $y_{i}$ and strong passivity of $\Xi \mathrm{im}$ plies $\alpha_{i}>0$. Augmenting the one-dimensional center-unstable dynamics (26) with the adaptation dynamics $\tau \dot{z}=-z+y_{i}$ and the outer loop $w_{i}=-z, w_{j}=0$ for $j \neq i$, we obtain

$$
\begin{aligned}
\dot{y}_{i} & =\epsilon y_{i}-\kappa^{\prime} y_{i}^{3}-\alpha_{i} z+\mathcal{O}\left(\left|\left(y_{i}, z\right)\right|^{4}\right) \quad \kappa^{\prime}>0, \alpha_{i}>0 \\
\dot{z} & =\delta\left(-z+y_{i}\right) \\
(\dot{\epsilon} & =0 \\
\dot{\delta} & =0) .
\end{aligned}
$$

Treating $\delta=\tau^{-1}$ as a state variable makes the adaptation equation part of the center-unstable manifold dynamics, locally defined around $\left(y_{i}, z, \epsilon, \delta\right)=(0,0,0,0)$ (see [29]). The equilibrium $\left(y_{i}, z\right)=(0,0)$ of (27) is stable for $\epsilon<\delta>0$ and unstable for $\epsilon>\delta>0$. Standard singular perturbation arguments, see, e.g., [10, pp. 445-448], prove that there exists a constant $\bar{\epsilon}>0$ and a neighborhood $\mathcal{V}$ of the equilibrium $(X, z)=(0,0)$ of (27) such that for any fixed $\delta$ and $\epsilon$ such that $0<\delta \ll \epsilon<\bar{\epsilon}$, all solutions with initial condition in $\mathcal{V} \backslash\{0\}$ converge to a unique limit cycle. Because of the time-scale separation induced by $0<\delta \ll \epsilon$, this limit cycle corresponds to a relaxation oscillation.

The global part of the proof is similar to that of Theorem 2: For $\delta>0$ and $\epsilon=0$, the equilibrium $(X, z)=(0,0)$ is globally asymptotically stable because the augmented storage function $\mathcal{S}_{k^{*}}=\delta S_{k^{*}}+(1 / 2) z^{2}$ satisfies the dissipation inequality $\dot{\mathcal{S}}_{k^{*}}=\delta \dot{S}_{k^{*}}+\dot{z} z=-\delta Y^{T} \Phi(Y)-\delta y_{i} z+\delta z\left(-z+y_{i}\right) \leq$ $-\delta\left(Y^{T} \Phi(Y)+z^{2}\right)$ which is analogous to (9). Using ultimate boundedness of the augmented system, the same arguments as in proof of Theorem 2 may be used.

Remark 2: If the forward system $\Xi$ is linear, strongly passive and detectable and the repeated nonlinearity $\phi(\cdot)$ satisfies the assumptions of Section II-B and is monotone increasing, then ultimate boundedness follows from Remark 1 since the adaptation dynamics $\tau \dot{z}=-z+y_{i}$ is passive.

\section{PASSIVE OSCILLATORS}

We define a passive oscillator as a system that admits the feedback representation (3)-(5) with the assumptions of Section II-B and satisfies the two following conditions.

1) The feedback system satisfies the dissipation inequality

$$
\dot{S}_{k} \leq\left(k-k_{\text {passive }}^{*}\right) Y^{T} Y-Y^{T} \Phi(Y)+W^{T} Y
$$

where $S_{k}(X)$ represents the storage function of the feedback system and $k_{\text {passive }}^{*} \geq 0$ is the critical value of $k$ above which it loses passivity.

2) When unforced $(W=0)$, the feedback system possesses a global limit cycle, i.e., a stable limit cycle which attracts all solutions except those belonging to the stable manifold of the origin.

The first condition necessarily holds if we assume that the forward block $\Xi$ is strongly passive. In Theorems $2-4$, we provided sufficient conditions for the second condition to be satisfied as well.

In order to illustrate this definition, we consider a nontrivial example of a SISO passive oscillator of order 3 . Here $u$ (respectively, $y$ ) denotes the scalar external input (respectively, output) of the SISO passive oscillator.

Example 3: Consider the feedback system in Fig. 3 with the monotone nonlinearity $\phi(y)=y^{3}$ and the second-order transfer function

$$
H(s)=\frac{\tau s+\omega_{n}^{2}}{s^{2}+2 \zeta \omega_{n} s+\omega_{n}^{2}}
$$


which is passive if $2 \zeta \tau \geq \omega_{n}>0$. Calculations detailed in [18] show that the feedback system is passive for $k \leq k_{\text {passive }}^{*}$ with

$$
k_{\text {passive }}^{*}=\min \left(1,\left(2 \zeta-\frac{\omega_{n}}{\tau}\right) \frac{\omega_{n}}{\tau}\right)
$$

and that a bifurcation occurs at $k=k^{*}$, with $k^{*}$ being given by (30), as shown at the bottom of the page. In general, a passive system in positive feedback with a static gain $k$ loses passivity before losing stability, i.e., $k_{\text {passive }}^{*}<k^{*}$. This means that, except for particular parameters values for which $k_{\text {passive }}^{*}=k^{*}$, Theorem 2 does not apply. However, as shown in [18], for parameters values satisfying

$$
2 \frac{\omega_{n}}{\tau}\left(\zeta-\frac{\omega_{n}}{\tau}\right)<k^{*}<2 \zeta \frac{\omega_{n}}{\tau} .
$$

Theorem 3 can be used because the Zames-Falb multiplier

$$
M(s)=1-Z(s) \quad Z(s)=\frac{\frac{\omega_{n}^{2}}{\tau}-2 \zeta \omega_{n}+k^{*} \tau}{s+\frac{\omega_{n}^{2}}{\tau}}
$$

results in the passive transfer function

$$
R_{k^{*}}(s) M(s)=\tau \frac{s}{s^{2}+\Omega^{2}}
$$

with $\Omega=\sqrt{\tau+\omega_{n}^{2}\left(1-k^{*}\right)}$. The transfer function $R_{k^{*}}(s) M(s)$ has exactly two imaginary poles on the imaginary axis. We are thus in the Hopf bifurcation scenario described in Theorem 3.

Applying Theorem 3 for parameter values satisfying (31), a SISO passive oscillator is thus obtained when $k \gtrsim k^{*}$, i.e.,

1) the feedback system satisfies the dissipation inequality $\dot{S}_{k} \leq\left(k-k_{\text {passive }}^{*}\right) y^{2}-y^{4}+u y$;

2) when unforced (no external input is applied to the feedback system), it possesses a global limit cycle for $k \gtrsim k^{*}$.

The passive oscillators used in the examples of the next sections correspond to those introduced in Example 3 with the particular parameters values $\omega_{n}=1, \zeta=1.25$, and $\tau=2$. Using these parameters values in (29) and (30), we obtain $k_{\text {passive }}^{*}=k^{*}=1$. These particular parameter values thus allow to directly apply Case 2) of Theorem 2 (without further requiring multipliers). Other numerical examples which require the use of multipliers are provided in [18].

\section{INTERCONNECTIONS OF PASSIVE OSCILLATORS}

As we have seen in the proofs of Theorems 2-4, the external characterization of - possibly high-dimensional-passive oscillators by the dissipation inequality (9) plays a role both in the supercritical character of the bifurcation and in the preservation of global convergence properties beyond the bifurcation value $k^{*}$. We now show that this external characterization also plays an important role in the study of oscillations in networks of interconnected passive oscillators.
Consider $N$ SISO passive oscillators. We assume that the critical value $k_{\text {passive }}^{*}$ is the same for all the oscillators. The state-space model of oscillator $i \in\{1, \ldots, N\}$ is given by

$$
\Sigma_{i}\left\{\begin{array}{l}
\dot{x}_{i}=f_{i}\left(x_{i}\right)+g_{i}\left(x_{i}\right) \tilde{u}_{i}, x_{i} \in \mathbb{R}^{p}, \tilde{u}_{i} \in \mathbb{R} \\
y_{i}=h_{i}\left(x_{i}\right), y_{i} \in \mathbb{R}
\end{array}\right.
$$

with the feedback interconnection

$$
\tilde{u}_{i}=-\phi_{k}\left(y_{i}\right)+u_{i}
$$

and satisfies the dissipation inequality

$$
\dot{S}_{k, i} \leq\left(k-k_{\text {passive }}^{*}\right) y_{i}^{2}-y_{i} \phi\left(y_{i}\right)+u_{i} y_{i} .
$$

The $N$ systems $\Sigma_{i}$ define a MIMO system $\Sigma$ with input $\tilde{U}=\left(\tilde{u}_{1}, \ldots, \tilde{u}_{N}\right)^{T}$ and output $Y=\left(y_{1}, \ldots, y_{N}\right)^{T}$. Likewise, the $N$ oscillators define a MIMO system with input $U=\left(u_{1}, \ldots, u_{N}\right)^{T}$ and output $Y$. This MIMO system satisfies the dissipation inequality

$$
\dot{S}_{k} \leq\left(k-k_{\text {passive }}^{*}\right) Y^{T} Y-Y^{T} \Phi(Y)+Y^{T} U
$$

where $S_{k}=\sum_{i=1}^{N} S_{k, i}$.

In the MIMO feedback representation of the network given in Fig. 6, the coupling is regarded as an additional feedback defined by

$$
U=-F(Y)+W
$$

where $F(\cdot)$ represents the (nonlinear) input-output coupling between the oscillators and $W$ is the new external input of the interconnected network. $F(\cdot)$ is assumed to be a $\mathcal{C}^{1}$ function in $\mathbb{R}^{N}$ satisfying $F(0)=0$. As illustrated in Fig. 6, the interconnected network equivalently admits the Lure representation that we have used in Theorems 2-4. In this representation, the system $\Xi$ is regarded as $\Sigma$ with the feedback interconnection $\tilde{U}=-F(Y)+V$. If the network input-output coupling $F(\cdot)$ is passive, that is, if

$$
Y^{T} F(Y) \geq 0 \quad \forall Y \in \mathbb{R}^{N}
$$

then the MIMO system $\Xi$ in Fig. 6 is also (strongly) passive (being the feedback interconnection of two (MIMO) passive systems). Theorems 2-4 can then be used to predict the onset of global limit cycles in the interconnected system.

Remark 3: We note that the strong passivity and zero-state detectability assumptions of Theorems 2 and 3 hold for the network if they hold for each individual oscillator.

Regarding the bifurcation value $k^{*}$ and the dimension of the center manifold of the network at this bifurcation value, we have the following result for the case of networks of identical oscillators with linear and symmetric input-output coupling.

Proposition 1: Consider a network of $N$ identical passive oscillators (33)-(34) with linear, symmetric input-output coupling $U=-\Gamma Y$ where $\Gamma=\Gamma^{T}$. Let $k_{0} \in \mathbb{R}$ be the smallest shift such

$$
k^{*}=-\frac{\sqrt{\tau^{4}+2 \omega_{n}^{2} \tau^{3}+\omega_{n}^{3}\left(\omega_{n}-4 \zeta\right) \tau^{2}+4 \omega_{n}^{4}\left(1-\zeta \omega_{n}\right) \tau+4 \zeta^{2} \omega_{n}^{6}}}{2 \omega_{n}^{2} \tau}+\frac{\tau\left(\tau+\omega_{n}^{2}\right)+2 \zeta \omega_{n}^{3}}{2 \omega_{n}^{2} \tau} .
$$




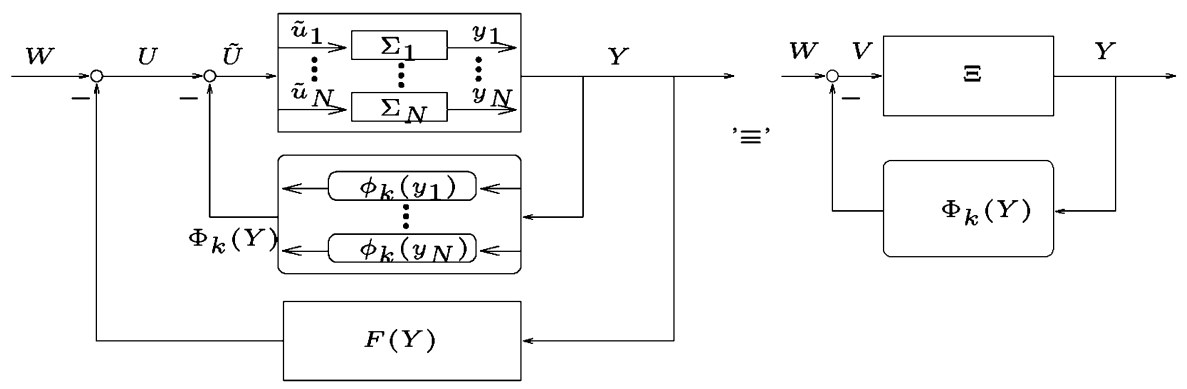

Fig. 6. MIMO representation of a network of SISO passive oscillators. Each block $\Sigma_{i}$ is (strongly) passive. $\Phi_{k}(Y)=\left(\phi_{k}\left(y_{1}\right), \ldots, \phi_{k}\left(y_{N}\right)\right)^{T}$ is a MIMO repeated nonlinearity. The repeated nonlinear element is $\phi_{k}(y)=-k y+\phi(y)$ where $\phi(\cdot)$ is a static nonlinear function that satisfies the assumptions of Section II-B. $F(Y)$ characterizes the network interconnection.

that $\Gamma^{\prime}=\Gamma^{\prime T}=\Gamma+k_{0} I_{N} \geq 0$ and $\operatorname{rank}\left(\Gamma^{\prime}\right)=N-1$. If each isolated passive oscillator has a center manifold of dimension two at $k=k_{\mathrm{osc}}^{*}$, then the network possesses a center manifold of the same dimension at the bifurcation value $k^{*}=k_{\mathrm{osc}}^{*}-k_{0}$.

Proof: Consider the Jacobian linearization of $\Sigma_{i}$ around the origin. Because all oscillators of the network are identical, they all share the same linearization. Let $G(s)$ be the transfer function associated to this shared linearized dynamics. The effect of the coupling appears in the linearization of the network dynamics through the additional term $\Gamma$. This is clearly seen in the expression of the poles of the transfer function $R_{k}(s)$ associated with the Jacobian linearization of the network dynamics around the origin. These poles may be calculated as the complex values of $s$ that lead to a rank drop for the MIMO transfer function

$$
\frac{1-k G(s)}{G(s)} I_{N}+\Gamma=\frac{1-\left(k+k_{0}\right) G(s)}{G(s)} I_{N}+\Gamma^{\prime} .
$$

Because $\Gamma^{\prime}=\Gamma^{\prime T} \geq 0$, there exists an orthogonal matrix $L$ such that $\Gamma^{\prime}=L \Lambda L^{T}$ with $\Lambda=\operatorname{diag}\left\{\lambda_{i}\right\}, i=1, \ldots, N$ where $\lambda_{i}$ denotes the $i$ th eigenvalue of the matrix $\Gamma^{\prime}$. Since $\Gamma^{\prime}$ has rank $N-1$, one can consider, without loss of generality, that $\lambda_{1}=0$ and $\lambda_{i}>0, i \in\{2, \ldots, N\}$. This implies that the smallest value of $k$ for which the matrix

$$
L\left(\frac{1-\left(k+k_{0}\right) G(s)}{G(s)} I_{N}+\Lambda\right) L^{T}
$$

loses rank is the one that leads to $1-\left(k+k_{0}\right) G(s)=0$. By assumption, this occurs for $k^{*}=k_{\mathrm{osc}}^{*}-k_{0}$. Moreover, from the preceding analysis it can be seen that the dimension of the center manifold at $k=k^{*}$ is equal to the dimension of the center manifold of one of the isolated, passive oscillators composing the network. This concludes the proof.

Based on Proposition 1 and on Theorems 2 and 3, we can directly extend the global limit cycle analysis of a single passive oscillator to a network of such identical passive oscillators. In the two examples that follow, we illustrate this result on some networks composed of an increasing number of identical passive oscillators. Each oscillator composing those networks examples is taken from Example 3 with the following parameters values $\omega_{n}=1, \zeta=1.25, \tau=2$. For these parameters values, using (29) and (30), we obtain $k_{\text {osc }}^{*}=k_{\text {passive }}^{*}=1$, which permits a direct application of Theorem 2.
Example 4: Consider the positive (respectively, negative) feedback coupling of 2 identical passive oscillators whose dynamics are given in Example 3. The resulting interconnection is illustrated in Fig. 7. The interconnection matrices are $\Gamma_{1}=\left(\begin{array}{cc}0 & -1 \\ -1 & 0\end{array}\right)$ and $\Gamma_{2}=\left(\begin{array}{ll}0 & 1 \\ 1 & 0\end{array}\right)$ respectively. The network is unchanged by the shifts $\Gamma^{\prime}=\Gamma+k_{0} I_{N}$ and $k^{\prime}=k+k_{0}$. In both cases, choosing $k_{0}=1$, the shifted matrices $\Gamma_{1}^{\prime}=$ $\left(\begin{array}{cc}1 & -1 \\ -1 & 1\end{array}\right)$ and $\Gamma_{2}^{\prime}=\left(\begin{array}{ll}1 & 1 \\ 1 & 1\end{array}\right)$ are positive semidefinite with rank 1. By Proposition 1 and the results presented in Example 3, the dimension of the center manifold is 2 and the assumptions of Theorem 2 are satisfied. The critical bifurcation value for the network is $k^{*}=k_{\text {osc }}^{*}-1$. From Theorem 2, we conclude that the network possesses a globally attractive limit cycle for $k \gtrsim k^{*}$. This is illustrated in Fig. 7 where simulation results for $k=0.3$ are presented. As can be seen on Fig. 7, the coupling defined by $\Gamma_{1}$ leads to a synchrone oscillation while the coupling defined by $\Gamma_{2}$ leads to an antiphase oscillation. We will return to the synchronization question in Section VIII.

Example 5: As an illustration of Theorem 2 for a network consisting of a large number of identical SISO passive oscillators, we consider a $S_{N}$ symmetry (all-to-all) network of passive oscillators. The dynamics of an isolated SISO passive oscillator is the one presented in Example 3. The linear $S_{N}$ symmetry coupling corresponds to the interconnection matrix $\Gamma=$ $\left[\gamma_{i, j}\right], i, j=1, \ldots, N$ with $\gamma_{i, i}=(N-1) K, \forall i \in\{1, \ldots, N\}$ and $\gamma_{i, j}=-K, \forall i \neq j$. In this matrix $\Gamma, K$ is a positive constant representing the coupling strength of the $S_{N}$ symmetry network. The eigenvalues of $\Gamma$ are $N K$ with a multiplicity $N-1$ and 0 . As a consequence, the rank of $\Gamma$ is $N-1$. By Proposition 1 and the results presented in Example 3, the dimension of the center manifold is 2 and the assumptions of Theorem 2 are satisfied. The critical bifurcation value for the network is $k^{*}=k_{\text {osc }}^{*}$. From Theorem 2, we conclude that the network possesses a globally attractive limit cycle for $k \gtrsim k^{*}$. This is illustrated by the simulation results presented in Fig. 8 for $k=2, N=5$, and $K=1$.

The same results hold for $D_{N}$ symmetry networks, i.e., bidirectional rings of oscillators. In the case of $D_{N}$ symmetry networks, the matrix $\Gamma$ has the form $\Gamma=\left[\gamma_{i, j}\right], i, j=1, \ldots, N$ with $\gamma_{i, i}=2 K, \gamma_{(i+1) \bmod (N), i}=\gamma_{i,(i+1) \bmod (N)}=-K, \forall i \in$ $\{1, \ldots, N\}$, and $\gamma_{i, j}=0$, for the other cases. This matrix is cyclic and its eigenvalues can be calculated analytically (see, e.g., [33]): $\lambda_{j}(\Gamma)=2 K(1-\cos (2 \pi j / N)) \geq 0, j=1, \ldots, N$. 


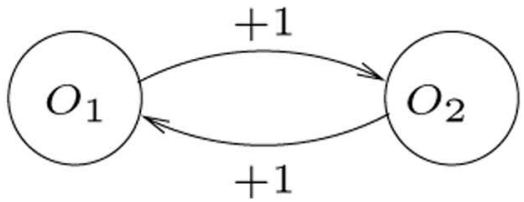

State-space of 2 oscillators tor $k_{1}=1, k_{p}=3.0000000-01$
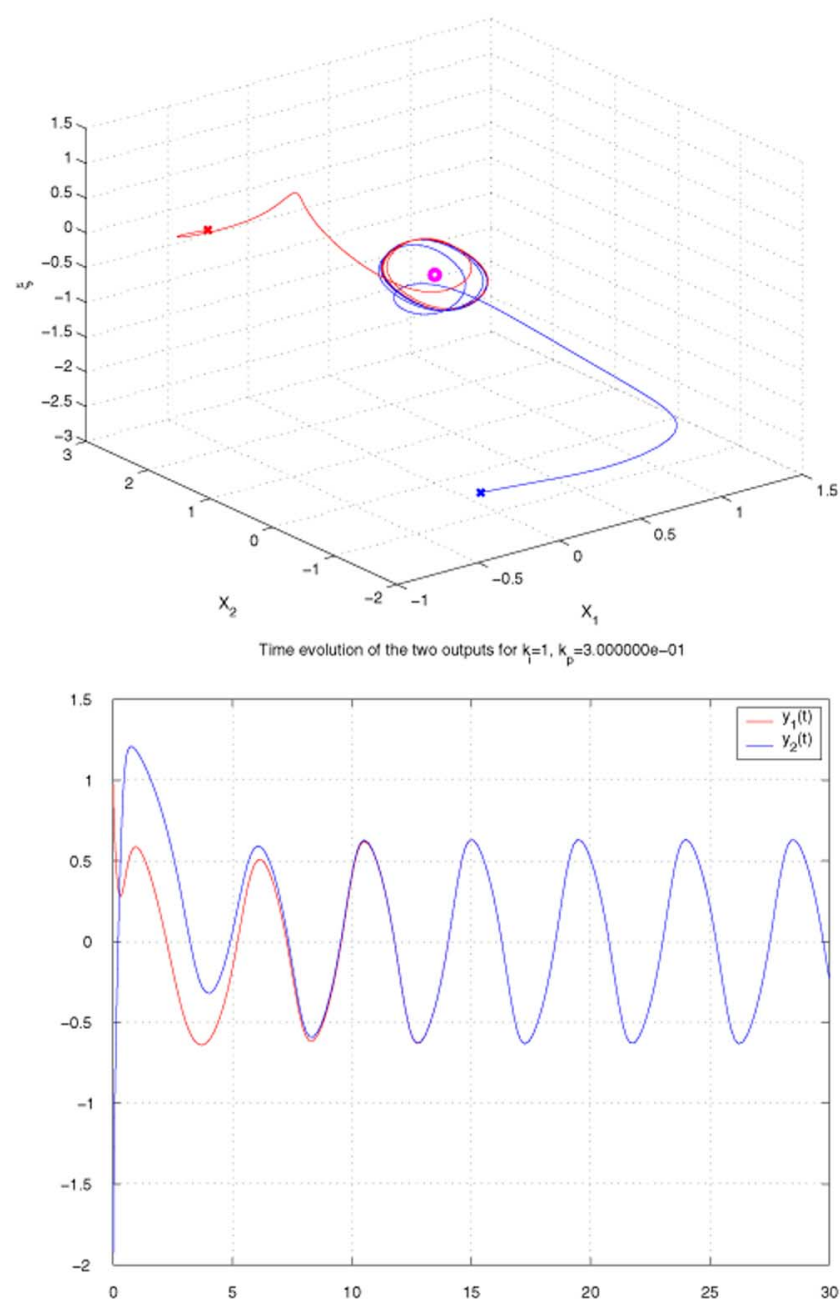

(a)

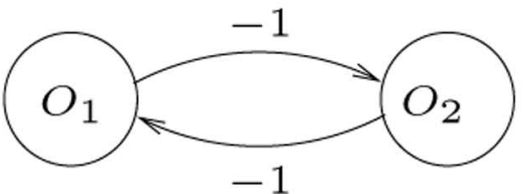

State-space of 2 oscillators tor $k_{1}=1, k_{p}=3.0000000-01$
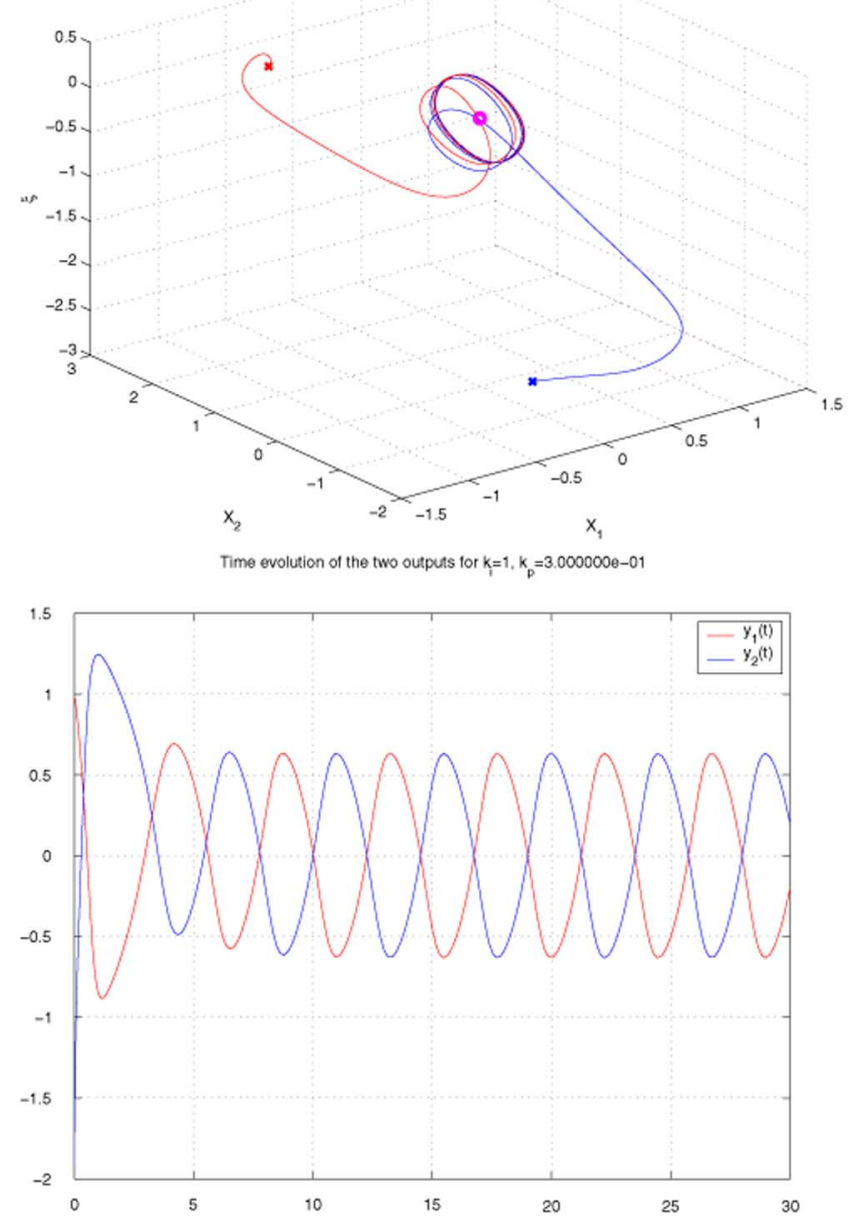

(b)

Fig. 7. Simulation results for a network of 2 identical passive oscillators. The circles represent the oscillators. Column (a) corresponds to $\Gamma_{1}$ and column (b) corresponds to $\Gamma_{2}$. Each oscillator is taken from Example 3 with the following parameters values $\omega_{n}=1, \zeta=1.25, \tau=2, k=0.3$. The critical bifurcation value for an isolated oscillator is $k_{\mathrm{osc}}^{*}=1$ and the corresponding bifurcation value for the network is $k^{*}=0$. The trajectories generated in the state-space of each oscillator are represented on the second row. A different color is used for each oscillator. The third row represents the time evolution of the outputs of the oscillators.

The rank of $\Gamma$ is once again equal to $N-1$ and the results of Theorem 2 may be directly applied.

\section{INCREMENTAL DISSIPATIVITY AND SYNCHRONIZATION}

Beyond the question of existence and (global) stability of sustained oscillations in a network of interconnected passive oscillators, an important issue concerns their relative oscillating behavior. The question of global synchronization among the oscillators is particularly relevant. Synchronization is a stability property for the difference between distinct solutions. Stability properties for the difference between solutions of a closed system are characterized by notions of incremental stability (see [34]-[36]). For open systems, the corresponding notion is incremental dissipativity.

\section{A. Incremental Dissipativity}

Consider an input-affine SISO system $\Upsilon$ represented by a state-space model of the form (3). We denote by $u(t), y(t)$ and $x(t)$, its input, output, and state, respectively. Let $x_{a}(t)$ and $x_{b}(t)$ be two solutions of $\Upsilon$, with the corresponding input-output pairs $\left(u_{a}(t), y_{a}(t)\right)$, and $\left(u_{b}(t), y_{b}(t)\right)$. We further consider the incremental variables $\Delta x=x_{a}-x_{b}, \Delta u=u_{a}-u_{b}$, and $\Delta y=y_{a}-y_{b}$. The system is incrementally dissipative if there exists an incremental storage 


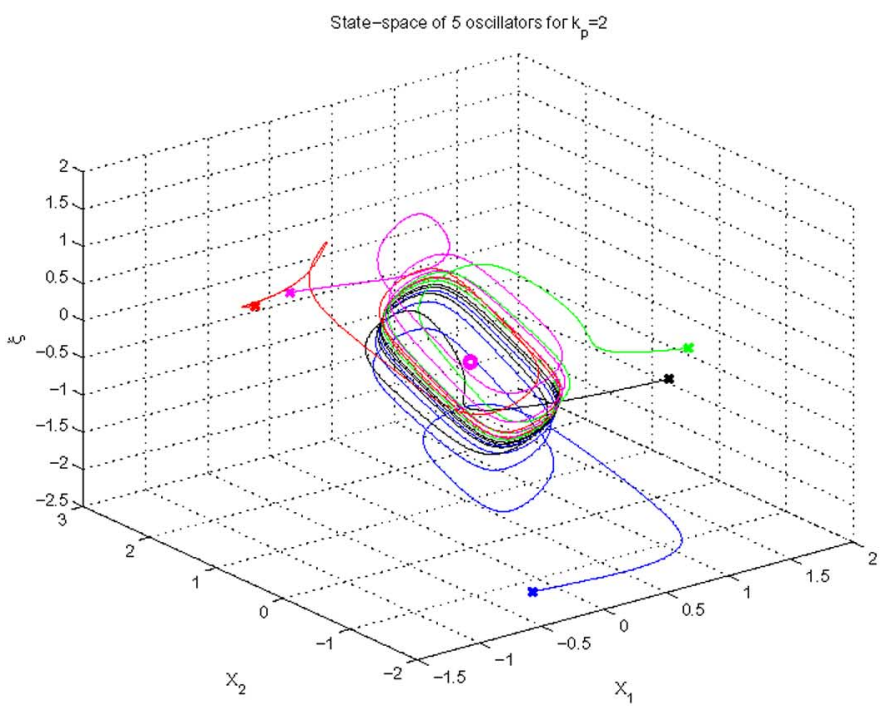

(a)

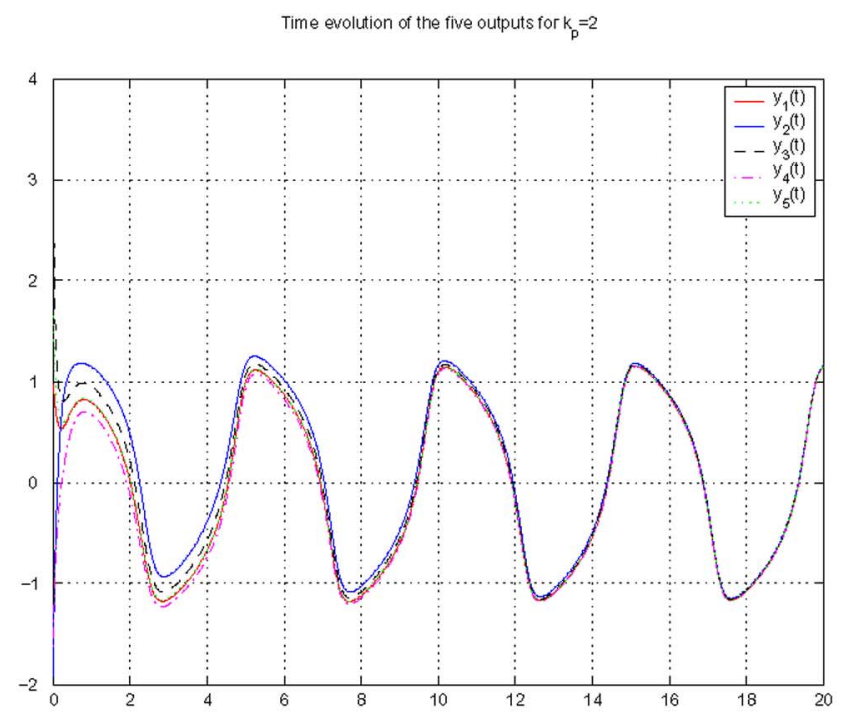

(b)

Fig. 8. Simulation results for a network of five identical passive oscillators coupled through $S_{5}$ symmetry. Each oscillator is taken from Example 3 with the following parameters values $\omega_{n}=1, \zeta=1.25, \tau=2, k=2$, and $K=1$. The critical bifurcation value for an isolated oscillator is $k_{\mathrm{osc}}^{*}=1$ and the corresponding bifurcation value for the network is $k^{*}=1$.

function $S_{\Delta}(\Delta x)$ and an incremental supply rate $\bar{w}(\Delta u, \Delta y)$ such that

$$
S_{\Delta}\left(\Delta x\left(T^{*}\right)\right)-S_{\Delta}(\Delta x(0)) \leq \int_{0}^{T^{*}} \bar{w}(\Delta u(t), \Delta y(t)) d t
$$

is satisfied for all $T^{*}>0$ and along any pair of trajectories $\left(x_{a}(t), x_{b}(t)\right)$. Incremental dissipativity (39) with the incremental supply rate $\bar{w}(\Delta u, \Delta y)=(\Delta u)^{T} \Delta y$ is called incremental passivity.

Passivity implies incremental passivity for linear systems, that is, if the quadratic storage $S(x)=x^{T} P x \geq 0$ satisfies the dissipation inequality $\dot{S} \leq u^{T} y$ then the incremental storage $S_{\Delta}(\Delta x)=(\Delta x)^{T} P \Delta x \geq 0$ satisfies the incremental dissipation inequality $\dot{S}_{\Delta} \leq(\Delta u)^{T} \Delta y$. Passivity also implies incremental passivity for monotone increasing, static nonlinearity: if $\phi(\cdot)$ is monotone increasing, then $\left(s_{1}-s_{2}\right)\left(\phi\left(s_{1}\right)-\phi\left(s_{2}\right)\right)=$ $\Delta s \Delta \phi(s) \geq \Delta s \psi(\Delta s) \geq 0, \forall \Delta s=s_{1}-s_{2}$, for some static nonlinearity $\psi(\cdot)$.

SISO passive oscillators made of the unforced feedback interconnection of a linear passive system $\Upsilon$ with a monotone increasing nonlinearity $\phi(\cdot)$ are thus also incrementally passive. In the following sections we restrict ourselves to Lure feedback systems made of the feedback interconnection of a linear passive system with a nonlinearity $\phi(\cdot)$ that is monotone increasing.

\section{B. Synchronization in Networks of Incrementally Passive Oscillators}

Consider a network of $N$ identical, SISO, incrementally passive oscillators. We assume that the only nonlinearity in each oscillator is due to the nonlinear function $\phi(\cdot)$ appearing in the definition of $\phi_{k}(\cdot)$. The dynamics for oscillator $i \in\{1, \ldots, N\}$ thus write

$$
\left\{\begin{array}{l}
\dot{x}_{i}=A x_{i}-B \phi_{k}\left(y_{i}\right)+B u_{i} \\
y_{i}=C x_{i}
\end{array}\right.
$$

where $u_{i} \in \mathbb{R}$ represents the external input of oscillator $i, y_{i} \in \mathbb{R}$ its output, and $x_{i} \in \mathbb{R}^{p}$ its state vector.

We assume linear input-output coupling between the SISO incrementally passive oscillators:

$$
U=-\Gamma Y
$$

We denote by $\lambda_{2}\left(\Gamma_{s}\right)$ the second smallest eigenvalue of $\Gamma_{s}$, with $\Gamma_{s}$ denoting the symmetric part of $\Gamma$, i.e., $\Gamma_{s}=\left(\Gamma+\Gamma^{T} / 2\right)$. Theorem 5 summaries the global synchronization conditions required in a network of identical, incrementally passive oscillators.

Theorem 5: Consider the linear interconnection (41) of $N$ identical, incrementally passive oscillators (40). Assume that $(A, C)$ is observable, $\phi(\cdot)$ is monotone increasing and each isolated oscillator $\left(u_{i} \equiv 0\right)$ possesses a globally asymptotically stable limit cycle in $\mathbb{R}^{p} \backslash E_{s}(0)$ where $E_{s}(0)$ denotes the stable manifold of the origin. If the interconnection matrix $\Gamma$ is a real, positive semidefinite matrix of rank $N-1$ such that $\Gamma \mathbf{1}=\Gamma^{T} \mathbf{1}=0$ then for $\lambda_{2}\left(\Gamma_{s}\right)>k-k_{\text {passive }}^{*}>0$ (strong coupling), the network has a limit cycle which attracts all solutions except those belonging to the stable manifold of the origin, and all the oscillators of the network exponentially synchronize.

Proof: Defining $\Pi=I_{N}-(1 / N) \mathbf{1 1}^{T}$, and denoting by $\Pi_{i}$ the $i$ th row of $\Pi$, the increment vector $\Pi_{i} Y$ measures the difference between the output of oscillator $i$ in the network and the average output $(1 / N) \sum_{i=1}^{N} y_{i}$. Let $S_{i}(x)=(1 / 2) x_{i}^{T} P x_{i}$ with $P=P^{T}>0$ be the storage function of oscillator $i$ and $X=\left(x_{1}^{T}, \ldots, x_{N}^{T}\right)^{T} \in \mathbb{R}^{p N}$ be the state vector associated to 
the network dynamics. For the interconnected system we consider the storage

$$
S_{\Delta}=\frac{1}{2}\left(\left(\Pi \otimes I_{p}\right) X\right)^{T}\left(I_{N} \otimes P\right)\left(\left(\Pi \otimes I_{p}\right) X\right)
$$

where ' $\otimes$ ' denotes the Kronecker product. $S_{\Delta}$ satisfies the dissipation inequality

$$
\begin{aligned}
\dot{S}_{\Delta} \leq\left(k-k_{\text {passive }}^{*}\right)(\Pi Y)^{T} \Pi Y-(\Pi Y)^{T} \Pi \Phi(Y) \\
+(\Pi Y)^{T} \Pi U .
\end{aligned}
$$

Because $\Pi$ is a projector, i.e., $\Pi^{2}=\Pi$, we have $(\Pi Y)^{T} \Pi \Phi(Y)=Y^{T} \Pi \Phi(Y)$. Moreover, $Y^{T} \Pi \Phi(Y)$ is nonnegative because $\Pi \Phi$ is a positive operator (see [37, Th. 3.10]). The dissipation inequality (42) thus implies

$$
\dot{S}_{\Delta} \leq\left(k-k_{\text {passive }}^{*}\right)(\Pi Y)^{T} \Pi Y+(\Pi Y)^{T} \Pi U
$$

Because $U=-\Gamma Y, \Pi \Gamma=\Gamma \Pi$ and $z^{T} \Gamma z=z^{T} \Gamma_{s} z, \forall z \in \mathbb{R}^{N}$, we obtain

$$
\dot{S}_{\Delta} \leq\left(k-k_{\text {passive }}^{*}\right)(\Pi Y)^{T} \Pi Y-(\Pi Y)^{T} \Gamma_{s} \Pi Y .
$$

The assumptions on $\Gamma$ imply that $\Gamma_{s}$ has rank $N-1$ and satisfies $\Gamma_{s} 1=0$, so that

$$
(\Pi Y)^{T} \Gamma_{s} \Pi Y \geq \lambda_{2}\left(\Gamma_{s}\right)(\Pi Y)^{T} \Pi Y
$$

which allows to rewrite (43) as

$$
\dot{S}_{\Delta} \leq\left(k-k_{\text {passive }}^{*}-\lambda_{2}\left(\Gamma_{s}\right)\right)|\Pi Y|^{2} .
$$

The strong coupling assumption implies

$$
\gamma=\lambda_{2}\left(\Gamma_{s}\right)-\left(k-k_{\text {passive }}^{*}\right)>0 .
$$

Integrating (44) over $\left[t_{0}, t_{0}+T^{*}\right]$ where $T^{*}>0$ is arbitrarily chosen, we obtain

$$
\begin{aligned}
\int_{t_{0}}^{t_{0}+T^{*}} \dot{S}_{\Delta} d \tau & \leq-\gamma \int_{t_{0}}^{t_{0}+T^{*}}|\Pi Y(\tau)|^{2} d \tau \\
& \leq-\alpha \gamma\left|\left(\Pi \otimes I_{p}\right) X\left(t_{0}\right)\right|^{2}, \alpha>0
\end{aligned}
$$

for all $\left(\Pi \otimes I_{p}\right) X\left(t_{0}\right), t_{0} \geq 0$. The last inequality comes from the observability of the pair $(A, C)$. Global exponential convergence of $\left(\Pi \otimes I_{p}\right) X$ to zero is then deduced from classical exponential stability theorems (see, for example, [38, Th. 1.5.2]). It implies that all solutions of the network exponentially converge to the invariant subspace

$$
\left\{X \in \mathbb{R}^{p N}: x_{1}=\cdots=x_{N}=\frac{\sum_{i=1}^{N} x_{i}}{N}\right\}
$$

that is, they exponentially synchronize. Since $\Gamma 1=0$, the dynamics of the network decouple in the invariant subspace (47), that is each oscillator behaves as if it was isolated, i.e., as if its dynamics were (40) with $u_{i}=0$. As a consequence all bounded solutions converge to the $\omega$-limit sets of the decoupled system. On the other hand, ultimate boundedness of the solutions follows from a MIMO generalization of the result in [20] (as discussed in Remark 1). We conclude that all solutions of the network converge to the $\omega$-limit sets of the uncoupled dynamics which correspond to the dynamics of an isolated oscillator, repeated $N$ times. This implies that all solutions, except those belonging to the stable manifold of the origin of the network, exponentially synchronize and converge towards a unique limit cycle.

Remark 4: The result still holds if the observability assumption on the pair $(A, C)$ is relaxed to a detectability assumption.

Remark 5: The global exponential stability result of $(\Pi \otimes$ $\left.I_{p}\right) X=0$ may also be viewed as an incremental input-to-state stability ( $\delta$-ISS) property of the network with $S_{\Delta}$ being the corresponding $\delta$-ISS Lyapunov function (see [34]).

Remark 6: Theorem 5 is closely linked to recent synchronization results presented in [39] and [40]. This may easily be noticed from the normal form of passive systems. The normal form for oscillator $i$ of the network is (see [7])

$$
\begin{aligned}
& \left(\begin{array}{c}
\dot{z}_{i} \\
\dot{y}_{i}
\end{array}\right) \\
& =\left(\begin{array}{cc}
Q & e \\
f^{T} & g
\end{array}\right)\left(\begin{array}{c}
z_{i} \\
y_{i}
\end{array}\right)+\left(\begin{array}{c}
\mathbf{0} \\
C B
\end{array}\right)\left(k y_{i}-\phi\left(y_{i}\right)\right) \\
& \quad-\sum_{j=1}^{N} \gamma_{i j}\left(\begin{array}{cc}
0 & \mathbf{0}^{T} \\
\mathbf{0} & C B
\end{array}\right)\left(\left(\begin{array}{c}
z_{j} \\
y_{j}
\end{array}\right)-\left(\begin{array}{c}
z_{i} \\
y_{i}
\end{array}\right)\right)
\end{aligned}
$$

where $C B$ is positive definite from the passivity assumption. Assume, as it is done by Slotine and Pogromsky, that $\gamma_{i j} \leq 0$ for $i \neq j$, and that $\gamma_{i i}=\sum_{j=1}^{N}\left|\gamma_{i j}\right|$. This implies that the couplings $-\gamma_{i j}\left(\begin{array}{cc}0 & \mathbf{0}^{T} \\ \mathbf{0} & C B\end{array}\right)$ are positive semidefinite. The symmetric part of the Jacobian of the uncoupled dynamics, divided according to the coupling structure, is given by

$$
J_{i s}=\left(\begin{array}{cc}
Q_{s} & \frac{1}{2}(e+f) \\
\frac{1}{2}(e+f)^{T} & g+C B k-C B \frac{d \phi\left(y_{i}\right)}{d y_{i}}
\end{array}\right) .
$$

It is then easily seen that the sufficient conditions given by Slotine in [39, Rem. 3 of Th. 2] are satisfied, i.e.,

1) $Q_{s}$ is contracting since it is Hurwitz from the passivity and detectability assumptions;

2) $\lambda_{\max }\left(g+C B k-C B\left(d \phi\left(y_{i}\right) / d y_{i}\right)\right)<g+C B k<\infty$ from the monotone increasing assumption;

3) $\sigma_{\max }((1 / 2)(e+f))=|(e+f / 2)|^{2}<\infty$.

Exploiting the special structure of passive oscillators, Theorem 5 additionally proves that the network solutions are bounded and that the network possesses a unique limit cycle which attracts (almost) all trajectories. 


\section{GRAPH INTERPRETATION OF THE INTERCONNECTION ASSUMPTIONS}

In this section, we give an interpretation of the interconnection assumptions of Proposition 1 and Theorem 5 in terms of directed graphs.

Consider a directed graph $\mathcal{G}$ with associated weighted adjacency matrix $A=\left[w_{i, j}\right], i, j=1, \ldots, N$ (see [41]). Assume that the graph is simple, i.e., $w_{i, j} \geq 0$ and $w_{i, i}=0, \forall i, j$. The corresponding weighted Laplacian matrix $\Gamma$ writes $\Gamma=$ $\left[\gamma_{i, j}\right], i, j=1, \ldots, N$ with $\gamma_{i, i}=\sum_{j \neq i}^{N} w_{i, j}, \forall i \in\{1, \ldots, N\}$ and $\gamma_{i, j}=-w_{i, j}, \forall i \neq j$. The interconnection rule $U=$ $-\Gamma Y$ then corresponds to the linear consensus protocol $u_{i}=$ $-\sum_{j=1}^{N} w_{i, j}\left(y_{i}-y_{j}\right)($ see [42]).

Proposition 1 and Theorem 5 require $\Gamma \geq 0$. This assumption holds if the graph is balanced, i.e., if $A \mathbf{1}=A^{T} \mathbf{1}$ (see [41]). This latter property implies $\Gamma \mathbf{1}=\Gamma^{T} \mathbf{1}(=0)$, which is a required assumption of Theorem 5 .

Proposition 1 and Theorem 5 require $\Gamma$ to have rank $N-$ 1 . This assumption holds provided that the graph is strongly connected (see [42]).

Finally, Proposition 1 requires $\Gamma$ to be symmetric, which is equivalent to assuming that the graph is undirected. In contrast, this assumption is not necessary for the synchronization result of Theorem 5.

Example 6: As an illustration of Theorem 5 for a nonsymmetric interconnection matrix, we consider a $Z_{N}$ symmetry network, i.e., a unidirectional ring of passive oscillators. The corresponding adjacency matrices writes $A=\left[w_{i, j}\right], i, j=1, \ldots, N$ with $w_{i,(i+1) \bmod (N)}=K, \forall i \in\{1, \ldots, N\}$ and $w_{i, j}=0$ in the other cases. The corresponding Laplacian matrix is $\Gamma=$ $\left[\gamma_{i, j}\right], i, j=1, \ldots, N$ with $i, i=K$ and $i,(i+1) \bmod (N)=$ $-K, \forall i \in\{1, \ldots, N\}$, and $i, j=0$ in the other cases. Because the graph is strongly connected and balanced, all assumptions of Theorem 5 hold and we can conclude to global, exponential synchronization towards a unique limit cycle for the network defined by this graph.

\section{CONClusion}

In this paper, we used dissipativity theory for the global analysis of limit cycles in a particular class of Lure dynamical systems called passive oscillators. The results of this paper are not restricted to low-dimensional passive oscillators and are wellsuited to the analysis of their input-output interconnections.

In particular, we have characterized two basic bifurcation scenarii in absolutely stable feedback systems. These bifurcation scenarii correspond to two global oscillation mechanisms generalizing the Van der Pol and Fitzhugh-Nagumo oscillators. The key assumption of the results is that the system is (strongly) passive at the bifurcation point (Theorem 2), an assumption that can be weakened by means of multiplier theory (Theorem 3). The consequence of that assumption is a specific dissipation inequality which is of interest for the global limit cycle analysis of the (isolated) feedback system as well as for the synchrony analysis of its interconnection with identical systems. In the analysis of interconnected oscillators, the assumptions on the interconnection have a natural interpretation in passivity theory as well as in graph theory.
Several important issues remain to be addressed in future work. In particular, a generalization of our theorems to the degenerate case when, at the critical bifurcation value, more than two eigenvalues simultaneously cross the imaginary axis would find applications, e.g., in Hamiltonian systems (see [18]). The robustness of the proposed analysis to interconnections of non identical oscillators is another important issue that deserves further research. Finally, the authors envision the application of the proposed approach to the analysis of possibly high-dimensional models of biochemical oscillators such as those found in [2].

\section{ACKNOWLEDGMENT}

The authors would like to thank the anonymous reviewers for their many suggestions for improving the quality of the manuscript. The first author would like to thank P.-A. Absil, R. Lambert, and J. Walmag for valuable comments and helpful discussions during various stages of this work.

\section{REFERENCES}

[1] A. Winfree, The Geometry of Biological Time, 2nd ed. New York: Springer-Verlag, 2000.

[2] A. Goldbeter, Biochemical Oscillations and Cellular Rhythms: The Molecular Bases of Periodic and Chaotic Behaviour. Cambridge, U.K.: Cambridge Univ. Press, 1996.

[3] S. H. Strogatz, Nonlinear Dynamics and Chaos. Cambridge, MA: Westview Press, 2000

[4] S. H. Strogatz, Sync: The Emerging Science of Spontaneous Order. New York: Hyperion, 2003.

[5] J. Willems, "Dissipative dynamical systems," Arch. Rat. Mech. Anal., vol. 45, pp. 321-393, 1972.

[6] A. van der Schaft, $\mathcal{L}_{2}$-Gain and Passivity Techniques in Nonlinear Control. London, U.K.: Springer-Verlag, 2000.

[7] R. Sepulchre, M. Jankovic, and P. Kokotovic, Constructive Nonlinear Control. London, U.K.: Springer-Verlag, 1997.

[8] L. O. Chua, "Passivity and complexity," IEEE Trans. Circuits Syst. I, vol. 46, no. 1, pp. 71-82, Jan. 1999.

[9] R. Sepulchre and G.-B. Stan, "Feedback mechanisms for global oscillations in Lure systems," Syst. Control Lett., vol. 54, no. 8, pp. 809-818, 2005.

[10] H. K. Khalil, Nonlinear Systems, 3rd ed. Upper Saddle River, NJ: Prentice-Hall, 2002.

[11] H. R. Wilson, Spikes, Decisions and Actions-Dynamical Foundations of Neuroscience. New York: Oxford Univ. Press, 1999.

[12] V. A. Yakubovich, "Frequency-domain criteria for oscillation in nonlinear systems with one stationary nonlinear component," Siberian Math. J., vol. 15, no. 5, pp. 1100-1129, 1973.

[13] E. A. Tomberg and V. A. Yakubovich, "Conditions for auto-oscillations in nonlinear systems," Siberian Math. J., vol. 30, no. 4, pp. 180-194, 1989.

[14] G. Leonov, I. Burkin, and A. Shepeljavyi, Frequency Methods in Oscillation Theory. Norwell, MA: Kluwer, 1996.

[15] A. Mees, Dynamics of Feedback Systems. New York: Wiley-Interscience, 1981.

[16] J. Goncalves, A. Megretski, and M. Dahleh, "Global analysis of piecewise linear systems using impact maps and quadratic surface Lyapunov functions," IEEE Trans. Autom. Control, vol. 48, no. 12, pp. 2089-2106, Dec. 2003.

[17] G.-B. Stan and R. Sepulchre, "Dissipativity and global analysis of limit cycles in networks of oscillators," in Proc. 16th Int. Symp. Mathematical Theory of Networks and Systems, Leuven, Belgium, 2004.

[18] G.-B. Stan, "Global analysis and synthesis of oscillations: A dissipativity approach" Ph.D. dissertation, Univ. Liège, Liège, Belgium, Mar. 2005 [Online]. Available: http://www.montefiore.ulg.ac.be/stan

[19] A. Graham, Kronecker Products and Matrix Calculus with Applica tions. New York: Ellis Horwood, 1981.

[20] M. Arcak and A. Teel, "Input-to-state stability for a class of Lurie systems," Automatica, vol. 38, no. 11, pp. 1945-1949, 2002.

[21] J. C. Willems, "Dissipative dynamical systems: Parts I and II," Arch Rat. Mech. Anal., vol. 45, pp. 321-393, 1972. 
[22] C. I. Byrnes, A. Isidori, and J. C. Willems, "Passivity, feedback equivalence, and global stabilization of minimum phase systems," IEEE Trans. Autom. Control, vol. 36, no. 1, pp. 1128-1240, Nov. 1991.

[23] G. Zames and P. L. Falb, "Stability conditions for systems with monotone and slope-restricted nonlinearities," SIAM J. Control Optim., vol. 6, no. 1, pp. 89-108, 1968.

[24] M. G. Safonov and V. V. Kulkarni, "Zames-Falb multipliers for MIMO nonlinearities," Int. J. Robust Nonlinear Control, vol. 10, pp. 1025-1038, 2000.

[25] A. Megretski and A. Rantzer, "System analysis via integral quadratic constraints," IEEE Trans. on Automatic Control, vol. 42, no. 6, pp. 819-830, Jun. 1997.

[26] V. M. Popov, "Absolute stability of nonlinear control systems of automatic control," Automation and Remote Control, vol. 22, pp. 857-875, 1962.

[27] H. K. Khalil, Nonlinear Systems, 2nd ed. Upper Saddle River, NJ: Prentice-Hall, 1996.

[28] L. Moreau and D. Aeyels, "Practical stability and stabilization," IEEE Trans. Autom. Control, vol. 45, no. 8, pp. 1554-1558, Aug. 2000.

[29] S. Wiggins, Introduction to Applied Nonlinear Dynamical Systems and Chaos. New York: Springer-Verlag, 1990, Texts in Applied Mathematics, 2.

[30] J. Carr, Applications of Center Manifold Theory. New York: Springer-Verlag, 1981.

[31] A. Isidori, Nonlinear Control Systems, 3rd ed. Berlin, Germany: Springer-Verlag, 1995.

[32] J. D. Murray, Mathematical Biology, I: An Introduction, 3rd ed. New York: Springer-Verlag, 2002.

[33] F. C. Hoppensteadt, An Introduction to the Mathematics of Neurons. Cambridge, U.K.: Cambridge Univ. Press, 1986.

[34] D. Angeli, "A Lyapunov approach to incremental stability properties," IEEE Trans. Autom. Control, vol. 47, no. 3, pp. 410-422, Mar. 2002.

[35] W. Lohmiller and J.-J. E. Slotine, "On contraction analysis for nonlinear systems," Automatica, vol. 34, no. 6, pp. 683-696, 1998.

[36] A. Pavlov, A. Pogromsky, N. van de Wouw, and H. Nijmeijer, "Convergent dynamics: A tribute to Boris Pavlovich Demidovich," Syst. Control Lett., vol. 52, pp. 257-261, 2004.

[37] J. Willems, The Analysis of Feedback Systems. Cambridge, MA: MIT Press, 1971

[38] S. Sastry and M. Bodson, Adaptive Control: Stability, Convergence, and Robustness. Upper Saddle River, NJ: Prentice-Hall, 1989.
[39] J.-J. Slotine and W. Wang, "A study of synchronization and group cooperation using partial contraction theory," in Block Island Workshop on Cooperative Control, K. V., Ed. New York: Springer-Verlag, 2003.

[40] A. Pogromsky, "Passivity based desing of synchronizing systems," Int. J. Bifur. Chaos, vol. 8, pp. 295-319, 1998.

[41] L. B. Cremean and R. M. Murray, "Stability analysis of interconnected nonlinear systems under matrix feedback," in Proc. 42nd Conf. Decision Control, Maui, HI, Dec. 2003, vol. 4, pp. 3078-3083, (Part One).

[42] R. Olfati-Saber and R. M. Murray, "Consensus problems in networks of agents with switching topology and time-delays," IEEE Trans. Autom. Control, vol. 49, no. 9, pp. 1520-1533, Sep. 2004.

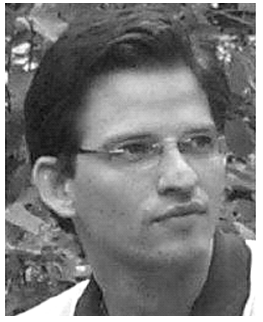

Guy-Bart Stan (M'04) received the Eng. and $\mathrm{Ph}$.D. degrees in applied sciences from the University of Liège, Liège, Belgium, in 2000 and 2005, respectively.

After working at Philips Applied Technologies as a Senior DSP Engineer in 2005, he moved to the University of Cambridge, Cambridge, U.K., where he is currently a Research Associate in the Engineering Department. His main research interests are in the fields of nonlinear systems analysis and control, systems biology, and bioinformatics.

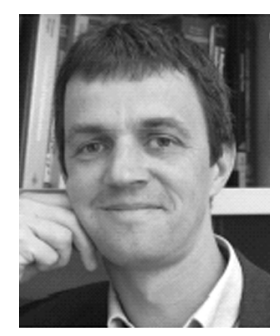

Rodolphe Sepulchre received the Eng. and Ph.D. degrees in applied mathematics from the University of Louvain, Louvain, Belgium, in 1990 and 1994, respectively.

He is a Professor in the Department of Electrical Engineering and Computer Science at the University of Liège, Liège, Belgium. From 1994 to 1996, he held a postdoctoral position at the University of California, Santa Barbara. In 2002-2003, he held a visiting position at Princeton University, Princeton, NJ. His research focuses on theory and applications of nonlinear dynamical systems. He is a coauthor of the book Constructive Nonlinear Control (New York: Springer-Verlag, 1997).

Dr. Sepulchre serves as an Associate Editor for the journal Mathematics of Control, Signals, and Systems. 